\title{
Singular moduli of higher level and special cycles
}

\author{
Stephan Ehlen ${ }^{*}$
}

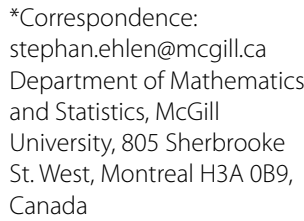

\begin{abstract}
We describe the complex multiplication (CM) values of modular functions for $\Gamma_{0}(N)$ whose divisor is given by a linear combination of Heegner divisors in terms of special cycles on the stack of CM elliptic curves. In particular, our results apply to Borcherds products of weight 0 for $\Gamma_{0}(N)$. By working out explicit formulas for the special cycles, we obtain the prime ideal factorizations of such CM values.
\end{abstract}

Keywords: Complex multiplication, CM values, Special cycles, Borcherds products Mathematics Subject Classification: Primary 11F03, Secondary 11G15

\section{Background}

Let $d<0$ be a negative fundamental discriminant and denote by $\mathcal{Q}_{d}$ the set of (positive and negative definite) integral binary quadratic forms of discriminant $d$. For every $Q \in \mathcal{Q}_{d}$ given by $Q(x, y)=a x^{2}+b x y+c y^{2}$ the unique root of $Q(\tau, 1)=0$ in $\mathbb{H}$, the complex upper half-plane, is denoted $\alpha_{Q}$. These points are called complex multiplication (CM) points because the associated elliptic curve has complex multiplication. That is, its endomorphism ring is an (in our case the maximal) order in an imaginary quadratic field. The values of $j(\tau)$ of the $j$-invariant at CM points are classically called singular moduli $[1,2]$. For $Q \in \mathcal{Q}_{d}$, the value $j\left(\alpha_{Q}\right)$ is an algebraic integer which generates the Hilbert class field $H$ of $k_{d}=\mathbb{Q}(\sqrt{d})$.

In their seminal article, Gross and Zagier [3] consider the modular function of level one

$$
\Psi(z, d)=\prod_{Q \in \mathrm{SL}_{2}(\mathbb{Z}) \backslash \mathcal{Q}_{d}}\left(j(z)-j\left(\alpha_{Q}\right)\right)^{1 / w_{d}},
$$

for $z \in \mathbb{H}$ and where $w_{d}$ is the number of roots of unity in $k_{d}$. They proved that for a negative fundamental discriminant $D$ coprime to $d$, we have

$$
\prod_{Q \in \mathrm{SL}_{2}(\mathbb{Z}) \backslash \mathcal{Q}_{D}} \Psi\left(\alpha_{Q}, d\right)^{2 / w_{D}}= \pm \prod_{\substack{x \in \mathbb{Z}, n, n^{\prime}>0 \\ 4 n n^{\prime}=d D-x^{2}}} n^{\epsilon\left(n^{\prime}\right)},
$$

where $\epsilon\left(n^{\prime}\right)= \pm 1$ is given explicitly in [3].

Gross and Zagier give an analytic and an algebraic proof of (1). The latter is given for prime discriminants only, but does in fact provide the prime ideal valuations of

(c) 2015 Ehlen. This article is distributed under the terms of the Creative Commons Attribution 4.0 International License (http:// creativecommons.org/licenses/by/4.0/), which permits unrestricted use, distribution, and reproduction in any medium, provided you give appropriate credit to the original author(s) and the source, provide a link to the Creative Commons license, and indicate if changes were made. 
the values $\Psi\left(\alpha_{Q}, d\right)$. Dorman [4] generalized this result to fundamental discriminants. Moreover, the contributions at the finite places to the height pairing studied in [5] essentially provide a generalization to higher level.

\section{Statement of results}

In this paper, we refine results of Bruinier and Yang [6] to relate the prime factorization of special values of certain modular functions to special cycles on the stack of CM elliptic curves. This has been used in $[7,8]$ to relate the coefficients of harmonic weak Maaß forms of weight one to these special cycles and to study the modularity of related arithmetic generating series. We use these results to determine the prime ideal factorization of such modular functions explicitly. In "Borcherds products" we show that we can apply our results to Borcherds products of weight zero.

We now define the higher level singular moduli we are considering. Let $d$ be a negative integer congruent to a square modulo $4 N$ and let $\mathcal{Q}_{d, N, r}$ be the set of integral binary quadratic forms $[a, b, c]=a x^{2}+b x y+c y^{2}$ of discriminant $d=b^{2}-4 a c$ such that $a$ is congruent to 0 modulo $N$ and $b$ is congruent to $r$ modulo $2 N$. For each form $Q=[a, b, c] \in \mathcal{Q}_{d, N, r}$ there is an associated CM point $\alpha_{Q}=\frac{-b+\sqrt{d}}{2 a}$ in $\mathbb{H}$. The group $\Gamma_{0}(N)$ acts on $\mathcal{Q}_{d, N, r}$ with finitely many orbits. We define the Heegner divisor $Z(d, r)$ to be the $\Gamma_{0}(N)$-invariant divisor (with rational coefficients)

$$
Z(d, r)=\sum_{Q \in \mathcal{Q}_{d, N, r}} \frac{\alpha_{Q}}{w_{Q}}
$$

where $w_{Q}$ is the order of the stabilizer of $Q$ in $\Gamma_{0}(N)$. We also denote the image of $Z(d, r)$ in $Y_{0}(N)=\Gamma_{0}(N) \backslash \mathbb{H}$ and its compactification $X_{0}(N)$ in the same way.

Now fix a square-free negative fundamental discriminant $D$ and let $H$ be the Hilbert class field of the imaginary quadratic field $k=k_{D}$ of discriminant $D$. We will write $\mathcal{O} \subset k$ for the ring of integers in $k$ and $\mathrm{N}(\cdot)=\mathrm{N}_{k / \mathbb{Q}}(\cdot)$ for the norm of $k / \mathbb{Q}$. The ring of integers in $H$ is denoted $\mathcal{O}_{H}$. We fix an embedding of $H$ into $\mathbb{C}$ and for $\rho \in \mathbb{Z}$ with $\rho^{2} \equiv D \bmod 4 N$ the integral ideal

$$
\mathfrak{n}=\left(N, \frac{\rho+\sqrt{D}}{2}\right)
$$

of norm $N$ in $k$. Consider the Heegner point $z_{D, \rho}$ contained in $Z(D, \rho)$ given by

$$
z_{D, \rho}=\frac{\rho+\sqrt{D}}{2 N} \in \mathbb{H} .
$$

Representatives for the remaining points in $Z(D, \rho)$ can be obtained as follows: if $\mathfrak{a}$ is an integral ideal of $k$, then $\mathfrak{a n}$ is an ideal of norm divisible by $N$. If we let $\alpha, \beta$ be generators of $\mathfrak{a n}$, such that $\alpha=\mathrm{N}(\mathfrak{a}) N$, then the point $\beta / \alpha$ lies in $\mathbb{H}$ and as the class [a] runs through the ideal class group of $k$, these points run through half of a system of representatives of $Z(D, \rho)$. The other half is obtained by also considering $-\bar{z}$ for each of these points.

We will now give a simpler version of our main result, Theorem 5.4, for prime level. Let $p$ be a prime and consider a meromorphic modular function $f$ on $Y_{0}(p)$. We assume 
in the introduction that $f$ is invariant under the Fricke involution and has a divisor on $\mathbb{H}$ of the form

$$
\operatorname{div}(f)=\sum_{r \bmod 2 \mathrm{p}} \sum_{\substack{d \in \mathbb{Z}_{<0} \\ d \equiv r^{2} \bmod 4 p}} c(d, r) Z(d, r) .
$$

Moreover, we assume that $\operatorname{div}(f)$ and $Z(D, \rho)$ have no common points and that the Fourier coefficients of $f$ are coprime integers. In particular, we have that $f \in \mathbb{Q}(j(\tau), j(p \tau))$. By the theory of complex multiplication, the value $f\left(z_{D, \rho}\right)$ is contained in the Hilbert class field $H$. The following theorem gives the multiplicity of a prime of the Hilbert class field in the prime ideal factorization of $f\left(z_{D, \rho}\right)$ in terms of certain special cycles $\mathcal{Z}(m, \mathfrak{n}, \mu)$ on $\operatorname{Spec} \mathcal{O}_{H}$ that parametrize elliptic curves with special endomorphisms of norm $m \mathrm{~N}(\mathfrak{n})$ satisfying a congruence condition given by $\mu \in \mathfrak{d}_{k}^{-1} \mathfrak{n} / \mathfrak{n}$ (see "Moduli of CM elliptic curves" and "Special endomorphisms" for definitions). Here, $\mathfrak{d}_{k}^{-1}$ denotes the inverse different of $k$. We write $\mathcal{Z}(m, \mathfrak{n}, \mu)$ as a formal sum

$$
\mathcal{Z}(m, \mathfrak{n}, \mu)=\sum_{\mathfrak{P}} \mathcal{Z}(m, \mathfrak{n}, \mu)_{\mathfrak{P}} \mathfrak{P}
$$

where the sum runs over all nonzero prime ideals of $H$ and $\mathcal{Z}(m, \mathfrak{n}, \mu)_{\mathfrak{P}} \in \mathbb{Q}$. These cycles are generalizations of those first considered by Kudla et al. [9] in connection with the coefficients of the derivative of an incoherent Eisenstein series of weight one.

Theorem 1 We have for every prime ideal $\mathfrak{P}$ of the Hilbert class field

$$
\operatorname{ord}_{\mathfrak{P}}\left(f\left(z_{D, \rho}\right)\right)=w_{D} \sum_{r \bmod 2 p} \sum_{d \in \mathbb{Q}_{<0}} c(d, r) \sum_{\substack{n \equiv \rho \cdot r \bmod 2 p \\ n^{2} \leq d D}} \mathcal{Z}\left(\frac{d D-n^{2}}{4 p|D|}, \mathfrak{n}, \frac{n+r \sqrt{D}}{2 \sqrt{D}}\right)_{\mathfrak{P}}
$$

Remark

(i) The set of CM values of $f$ at points in $Z(D, \rho)$ forms a single Galois orbit and the valuations of other points in $Z(D, \rho)$ can be obtained using Shimura reciprocity and Proposition 2 below.

(ii) Note that there exist modular functions whose divisor is given by a linear combination of Heegner divisors on $Y_{0}(N)$ that are not obtained as Borcherds products. This has been remarked by Borcherds [10, Example 5.2] and follows from Theorem 7.7 of [6]. We also remark that Bruinier [11] proved a converse theorem for Borcherds products which shows that the case of modular curves is quite exceptional in this regard.

To obtain formulas for the quantities in Theorem 5.4 or Theorem 1 above, we use Gross' formula for the length of the local rings of $\mathcal{Z}(m, \mathfrak{n}, \mu)$ as in [12], where the arithmetic degrees of these cycles have been computed. In the case of a prime discriminant these formulas are completely explicit and rather simple, as we shall see below. 
Fix a fractional ideal $\mathfrak{n} \subset k$ and let $\mu \in \mathfrak{d}_{k}^{-1} \mathfrak{n} / \mathfrak{n}$ and $m \in \mathbb{Q}_{>0}$. We write [a] for the class of the fractional ideal $\mathfrak{a}$ in the class group $\mathrm{Cl}_{k}$ of $k$. We define a set of rational primes by

$$
\operatorname{Diff}(m)=\left\{p<\infty \mid(-m \mathrm{~N}(\mathfrak{n}), D)_{p}=-1\right\}
$$

and for $n \in \mathbb{Z}$ and $[\mathfrak{b}] \in \mathrm{Cl}_{k}$ we let

$$
\rho(n,[\mathfrak{b}])=\#\{\mathfrak{a} \subset \mathcal{O} \mid \mathrm{N}(\mathfrak{a})=n, \mathfrak{a} \in[\mathfrak{b}]\} .
$$

If $m$ is a rational number and $p$ is a rational prime which is non-split in $k$, we define

$$
v_{p}(m)= \begin{cases}\frac{1}{2}\left(\operatorname{ord}_{p}(m)+1\right), & \text { if } p \text { is inert in } k \\ \left(\operatorname{ord}_{p}(m|D|)\right), & \text { if } p \text { is ramified in } k .\end{cases}
$$

We obtain the following result:

Proposition 2 Let $D=-$ for a prime $l \equiv 3 \bmod 4$.

(i) We have $\mathcal{Z}(m, \mathfrak{n}, \mu)_{\mathfrak{P}}=0$ unless $|\operatorname{Diff}(m)|=1$ and $m+\mathrm{N}(\mu) / \mathrm{N}(\mathfrak{n}) \in \mathbb{Z}$.

(ii) Assume that $\operatorname{Diff}(m)=\{p\}$ and that $m+\mathrm{N}(\mu) / \mathrm{N}(\mathfrak{n}) \in \mathbb{Z}$. There is a unique prime ideal $\mathfrak{P}_{0} \mid$ p fixed by complex conjugation, $\overline{\mathfrak{P}}_{0}=\mathfrak{P}_{0}$. For $\mathfrak{P}=\mathfrak{P}_{0}^{\sigma}$, where $\sigma=\sigma(\mathfrak{b})$ corresponds to the ideal class of $\mathfrak{b}$ under the Artin map $(\cdot, H / k)$, we have $\mathcal{Z}(m, \mathfrak{n}, \mu)_{\mathfrak{P}}=2^{o(m)-1} v_{p}(m) \rho\left(m|D| / p,\left[\mathfrak{n} \mathfrak{b}^{-2}\right]\right)$, where $o(m)=1$ if $\operatorname{ord}_{l}(m l)>0$ and $o(m)=0$, otherwise.

\section{Outline}

The paper is organized as follows: In "Preliminaries and notation", we set up the notation and give basic definitions. The moduli stack of CM elliptic curves is introduced in "Moduli of CM elliptic curves" and in "Special endomorphisms" we describe the special cycles and prove the explicit formulas for the multiplicities. The integral model for the modular curve $X_{0}(N)$ will be recalled in "An integral model for the modular curve" and in "CM values of modular functions" we prove the main result of this paper (Theorem 5.4). The applications to Borcherds products are discussed in "Borcherds products" and finally, "Some numerical examples" covers some numerical examples illustrating our results.

\section{Preliminaries and notation}

We write $\mathbb{Z}$ for the integers, $\mathbb{Q}$ for the field of rational numbers, $\mathbb{Z}_{p}$ for the ring of $p$-adic integers and $\mathbb{Q}_{p}$ for the field of $p$-adic numbers. We also use the notation $\hat{\mathbb{Z}}=\prod_{p} \mathbb{Z}_{p}$, where the product is over all primes. We write $\mathbb{A}$ for the adeles of $\mathbb{Q}$ and use the notation $\mathbb{A}_{f}$ for the finite adeles of $\mathbb{Q}$. If $k$ is a number field, we write $\mathcal{O}_{k}$ for its ring of integers and $\mathbb{A}_{k}$ (and $\mathbb{A}_{k, f}$ ) for the adeles (and finite adeles) of $k$. Moreover, we write $\hat{\mathcal{O}}_{k}$ for the tensor product $\mathcal{O}_{k} \otimes_{\mathbb{Z}} \hat{\mathbb{Z}}$.

\subsection{Modular forms}

We briefly recall the definition of modular forms for $\Gamma_{0}(N)$. We refer to one of the standard references for details, for instance [13-16].

Let $N$ be a positive integer and consider the congruence subgroup $\Gamma_{0}(N) \subset \mathrm{SL}_{2}(\mathbb{Z})$ defined by 


$$
\Gamma_{0}(N)=\left\{\left(\begin{array}{ll}
a & b \\
c & d
\end{array}\right) \in \mathrm{SL}_{2}(\mathbb{Z}) \mid c \equiv 0 \bmod N\right\} .
$$

For an integer $k \in \mathbb{Z}$ and a matrix $\gamma \in \mathrm{SL}_{2}(\mathbb{Z})$, the Petersson slash operator is defined on functions $f: \mathbb{H} \rightarrow \mathbb{C}$ by

$$
\left(\left.f\right|_{k} \gamma\right)(\tau)=(c \tau+d)^{-k} f(\gamma \tau), \quad \text { where } \gamma=\left(\begin{array}{ll}
a & b \\
c & d
\end{array}\right) \in \mathrm{SL}_{2}(\mathbb{Z}) .
$$

Definition 1.1 A meromorphic function $f: \mathbb{H} \rightarrow \mathbb{C}$ is called a meromorphic modular form of weight $k \in \mathbb{Z}$ for $\Gamma_{0}(N) \subset \mathrm{SL}_{2}(\mathbb{Z})$ if

(i) $\left(\left.f\right|_{k} \gamma\right)(\tau)=f(\tau)$ for all $\gamma \in \Gamma_{0}(N)$

(ii) and $f$ is meromorphic at the cusps of $\Gamma_{0}(N)$.

The conditions at the cusps can be phrased in terms of Fourier expansions. A meromorphic modular form $f$ has a Fourier expansion (at the cusp $\infty$ ) of the form

$$
f(\tau)=\sum_{n \gg-\infty}^{\infty} a_{f}(n) q^{n},
$$

where $q=e^{2 \pi i \tau}=e(\tau)$. That is, if $f$ is a meromorphic modular form, then there are only finitely many non-vanishing Fourier coefficients $a_{f}(n)$ with $n<0$. The conditions at the other cusps are similar. By a modular function for $\Gamma_{0}(N)$, we mean a meromorphic modular form of weight 0 for $\Gamma_{0}(N)$. It is well known [17] that the field of modular functions for $\Gamma_{0}(N)$ is generated by $j(\tau)$ and $j_{N}(\tau)=j(N \tau)$.

\subsection{Elliptic curves}

We recall the definition of an elliptic curve over an arbitrary base scheme. We refer to Chapter 2 of the book by Katz and Mazur [18] and the two books by Silverman [19, 20] for details.

Definition 1.2 Let $S$ be a scheme. A proper smooth curve $E \rightarrow S$ with geometrically connected fibers of genus one together with a section $0: S \rightarrow E$, is called an elliptic curve.

It is an important fact that an elliptic curve admits a unique structure as a group scheme [18], Theorem 2.1.2]. It is well known (see [19, Corollary 9.4] and [21]) that the endomorphism ring of an elliptic curve over a field is either $\mathbb{Z}$, an order in an imaginary quadratic field or an order in a quaternion algebra.

We will use the notation

$$
\mathbb{B}=\left(\frac{a, b}{F}\right),
$$

to denote the quaternion algebra $F \oplus F \alpha \oplus F \beta \oplus F \alpha \beta$ with $\alpha^{2}=a$ and $\beta^{2}=b$ and $\alpha \beta=-\beta \alpha$. We say that the quaternion algebra $\mathbb{B}$ is split over $F$ if $\mathbb{B}$ is isomorphic to $M_{2}(F)$. Here, $M_{2}(F)$ is the algebra of $2 \times 2$-matrices over $F$. 
For a quaternion algebra $\mathbb{B}$ over $\mathbb{Q}$, we say that $\mathbb{B}$ is split at a prime $p($ or $\infty)$, if $\mathbb{B} \otimes_{\mathbb{Q}} \mathbb{Q}_{p}$ is split over $\mathbb{Q}_{p}$. Otherwise, we say that $\mathbb{B}$ is ramified at $p($ or $\infty)$. We use the same convention over other fields.

Let $E / k$ be an elliptic curve over a field $k$. If the endomorphism ring $\operatorname{End}_{k}(E)$ contains an order $\mathcal{O} \subset k$ for an imaginary quadratic field $k$, then we say that $E$ has complex multiplication (CM) by $\mathcal{O}$.

A $C M$ elliptic curve over a scheme $S$ is a pair $(E, \iota)$ consisting of an elliptic curve $E / S$ and an action $\iota: \mathcal{O} \hookrightarrow \operatorname{End}_{S}(E)$ of an order $\mathcal{O}$ in an imaginary quadratic field on $\operatorname{End}_{S}(E)$.

If $\operatorname{End}_{S}(E)=\mathcal{O} \subset \mathbb{B}$ is an order in a quaternion algebra, then we say that $E$ is supersingular. Supersingular elliptic curves only occur in positive characteristic (cf. VI., Theorem 6.1 of [19]).

\subsection{The Hilbert class field}

We recall only very briefly some facts from class field theory that we need later, in particular the Artin map. We refer to [20, Chapter II] or [17] for details. Let $k$ be a totally imaginary field and let $L$ be a finite abelian extension of $k$. Write $\mathcal{O}_{L}$ for the ring of integers in $L$. Let $\mathfrak{p}$ be a prime ideal of $k$ that does not ramify in $L$. There is a unique element $\sigma_{\mathfrak{p}} \in \operatorname{Gal}(L / k)$, such that

$$
\sigma_{\mathfrak{p}}(x) \equiv x^{\mathrm{N}_{L / k}(\mathfrak{p})} \bmod \mathfrak{P}
$$

for all $x \in \mathcal{O}_{L}$ and any prime ideal $\mathfrak{P} \mid \mathfrak{p}$. If $\mathfrak{c}$ is an integral ideal of $k$ which is divisible by all primes that ramify in $L / k$ and $I(\mathfrak{c})$ is the group of fractional ideals that are relatively prime to $c$, then the Artin map is defined as

$$
(\cdot, L / k): I(\mathfrak{c}) \rightarrow \operatorname{Gal}(L / k)
$$

by extending the map $\sigma_{\mathfrak{p}}$ linearly.

We let $k$ be an imaginary quadratic field and $H$ be the Hilbert class field of $k$. This is the maximal unramified abelian extension of $k$. It corresponds to the ray class field for $\mathfrak{c}=1$. By class field theory, we obtain in this case [20, II, Example 3.3] an isomorphism via the Artin map

$$
(\cdot, H / k): \mathrm{Cl}_{\mathrm{k}} \rightarrow \mathrm{Gal}(H / k) \text {. }
$$

We will use the convention that we write $\sigma(\mathfrak{a})=\sigma([\mathfrak{a}])$ for $([\mathfrak{a}], H / k)$, the image of the (class of the) fractional ideal $\mathfrak{a}$ under this map.

\section{Moduli of CM elliptic curves}

In this section we recall some facts about the moduli stack of CM elliptic curves and special 0-cycles on it. These cycles will play an important role in the description of the values of modular functions on $Y_{0}(N)$.

Let $S$ be a scheme. By a scheme over $S$ or an $S$-scheme, we mean a scheme $X$ together with a morphism $\pi: X \rightarrow S$. We denote by $(\mathrm{Sch} / S)$ the category of schemes over $S$. The morphisms in this category are morphisms of $S$-schemes, that is, morphisms that are compatible with the given morphisms to $S$. Here and throughout, if $X$ is a stack over a base scheme $S$ and $T$ is an $S$-scheme, we abbreviate

$$
X_{/ T}=X \times_{S} T .
$$


If $T=\operatorname{Spec} R$ with $R$ a ring, we simply write $X_{/ R}$ for $X_{/ \operatorname{Spec} R}$.

Here and for the rest of this section, let $D$ be a negative fundamental discriminant and denote by $k=k_{D}$ the imaginary quadratic field of discriminant $D<0$. We write $\mathcal{O}_{D}$ for the ring of integers of $k$ and let $H=H_{D}$ be the Hilbert class field of $k$. We remark that our methods should also work for non-fundamental discriminants but some of the calculations would certainly get more involved.

We consider the moduli problem which assigns to a base scheme $S$ over $\mathcal{O}_{D}$ the category $C_{D}^{+}(S)$ of pairs $(E, \iota)$, where

(i) $E$ is an elliptic curve over $S$ with complex multiplication $\iota: \mathcal{O}_{D} \hookrightarrow \operatorname{End}(E)$,

(ii) such that the induced map

$$
\operatorname{Lie}(\iota): \mathcal{O}_{D} \rightarrow \operatorname{End}_{\mathcal{O}_{S}}(\operatorname{Lie} E)=\mathcal{O}_{S},
$$$$
\text { coincides with the structure map } S \rightarrow \operatorname{Spec} \mathcal{O}_{D} \text {. }
$$

The morphisms in this category are isomorphisms that are compatible with the actions.

Moreover, we denote by $C_{D}=\operatorname{cRes}_{\mathcal{O}_{D} / \mathbb{Z}}\left(C_{D}^{+}\right)$the restriction of coefficients of $C_{D}^{+}$to $\mathbb{Z}$ (in the sense of Grothendieck). That is, the structure map of $C_{D}$ is given by $C_{D}^{+} \rightarrow \operatorname{Spec} \mathcal{O}_{D} \rightarrow \operatorname{Spec} \mathbb{Z}$. This describes the moduli problem without the normalization (2).

Proposition 2.1 The moduli problem $C_{D}^{+}$is represented by an algebraic stack, also denoted by $C_{D}^{+}$, which is smooth of relative dimension 0 and proper over $\operatorname{Spec} \mathcal{O}_{D}$. If $R$ is a discrete valuation ring with algebraically closed residue field $\mathbb{F}$, the reduction map $C_{D}^{+}(R) \rightarrow C_{D}^{+}(\mathbb{F})$ is surjective. Consequently, $C_{D}$ is also represented by an algebraic stack of relative dimension 0 over $\operatorname{Spec} \mathbb{Z}$, which is finite (and thus proper).

Proof This is a consequence of the canonical lifting theorem [22, 23]. Properness follows from the fact that all points of $C_{D}$ in characteristic 0 have potentially good reduction and the valuative criterion of properness. See [9, Section 5] or [24] for details.

Lemma 2.2 The coarse moduli scheme $\mathrm{C}_{D}^{+}$of $C_{D}^{+}$is isomorphic to $\mathrm{Spec} \mathcal{O}_{H}$ as a scheme over $\mathcal{O}_{D}$. Consequently, the coarse moduli scheme $\mathbf{C}_{D}$ of $C_{D}$ is isomorphic to $\operatorname{Spec} \mathcal{O}_{H}$ as a scheme over $\mathbb{Z}$.

Proof This is [9, Corollary 5.4]. There, the authors prove the corresponding isomorphism for $C_{D}^{+}$, whose coarse moduli scheme is $\operatorname{Spec} \mathcal{O}_{H} \rightarrow \operatorname{Spec} \mathcal{O}_{D}$.

We denote by pr: $C_{D}^{+} \rightarrow \mathbf{C}_{D}^{+}$the canonical map to the coarse moduli scheme.

Proposition 2.3 Let $\xi \in C_{D}^{+}$be a geometric point and let $\operatorname{pr}(\xi)=\bar{\xi}$ be the corresponding point of $\mathbf{C}_{D}^{+}$. Then $\hat{\mathcal{O}}_{C_{D}^{+}, \xi}=\hat{\mathcal{O}}_{\mathbf{C}_{D}^{+}, \bar{\xi}}$ where $\hat{\mathcal{O}}_{C_{D}^{+}, \xi}$ and $\hat{\mathcal{O}}_{\mathbf{C}_{D}^{+}, \bar{\xi}}$ denote the completions of the étale local rings at $\xi$ and $\bar{\xi}$, respectively.

Proof This is Corollary 5.2 in [9]. 
We now describe the geometric points of $C_{D}$ in every characteristic. The following construction is very important for us:

Recall that over $\mathbb{C}$, we have a canonical bijection

$$
C_{D}^{+}(\mathbb{C}) \cong k^{\times} \backslash \mathbb{A}_{k, f}^{\times} / \hat{\mathcal{O}}_{D}^{\times}
$$

given by the theory of complex multiplication [20]. Here and throughout, we write $\mathbb{A}_{k, f}^{\times}$ for the finite ideles over $k$. To an idele $h \in \mathbb{A}_{k, f}^{\times}$that corresponds to the ideal class [ $(h)$ ], the bijection assigns the (isomorphism class of the) elliptic curve with complex points

$$
E(\mathbb{C})=\mathbb{C} /(h) .
$$

Moreover, if $(E, \iota) \in C_{D}^{+}(\mathbb{C})$ is given by $(\mathbb{C} / \Lambda, \iota)$, then multiplication with $h \in \mathbb{A}_{k, f}^{\times}$on the right-hand side of (3) corresponds to

$$
E \mapsto(h) \otimes_{\mathcal{O}_{D}} E,
$$

where $(h) \otimes_{\mathcal{O}_{D}} E$ is the elliptic curve over $\mathbb{C}$ with complex points

$$
\left((h) \otimes_{\mathcal{O}_{D}} E\right)(\mathbb{C}) \cong \mathbb{C} /(h) \Lambda .
$$

This defines an action of the class group $\mathrm{Cl}_{\mathrm{k}}$ on the set of isomorphism classes of $\mathrm{CM}$ elliptic curves (with $\mathrm{CM}$ by $\mathcal{O}_{D}$ ) over $\mathbb{C}$.

Now let $(E, \iota) \in C_{D}^{+}(S)$ for a scheme $S$ and let $h \in \mathbb{A}_{k, f}^{\times}$, corresponding to the ideal $(h)$. Then we can define a functor from the category of $S$-schemes to the category of $\mathcal{O}_{D}$ -modules by

$$
T \mapsto(h) \otimes_{\mathcal{O}_{D}} E(T) .
$$

This functor is in fact represented by an elliptic curve over $S$ and the construction is called the Serre construction. We denote the elliptic curve representing this functor by $h . E=(h) \otimes_{\mathcal{O}_{D}} E$. For details, the reader may consult [25] and [26, Section 7].

We follow the description given in [9] to describe the geometric points of $C_{D}^{+}$in positive characteristic.

Proposition 2.4 (Corollary 5.5 of [9]) Let $\mathfrak{p}$ be a prime ideal of $k$ and let $\overline{\kappa(\mathfrak{p})}$ denote an algebraic closure of the residue field $\kappa(\mathfrak{p})$. We have a bijection

$$
C_{D}^{+}(\overline{\kappa(\mathfrak{p})}) \cong k^{\times} \backslash \mathbb{A}_{k, f}^{\times} / \hat{\mathcal{O}}_{D}^{\times}
$$

The action by the Frobenius automorphism over $\kappa(\mathfrak{p})$ on the left-hand side corresponds to the translation by an idele of the form $(1, \ldots, 1, \pi, 1, \ldots)$, where $\pi$ is a uniformizer at $\mathfrak{p}$.

The proposition establishes a simply transitive action of the class group $\mathrm{Cl}_{\mathrm{k}}$ on the points $C_{D}^{+}(\overline{\mathbb{F}})$ over any algebraically closed field $\overline{\mathbb{F}}$. We also have a bijection on geometric points $C_{D}^{+}(\overline{\mathbb{F}}) \cong \operatorname{Spec} \mathcal{O}_{H}(\overline{\mathbb{F}})$. On the points Spec $\mathcal{O}_{H}(\overline{\mathbb{F}})$, we have an action of the Galois $\operatorname{group} \operatorname{Gal}(H / k)$.

Fix a morphism pr: $C_{D}^{+} \rightarrow \operatorname{Spec} \mathcal{O}_{H}$. Then pr induces an isomorphism

$$
\operatorname{pr}_{\mathbb{F}}: C_{D}^{+}(\overline{\mathbb{F}}) \cong \operatorname{Spec} \mathcal{O}_{H}(\overline{\mathbb{F}})
$$


on geometric points over any algebraically closed field $\overline{\mathbb{F}}$. For $\overline{\mathbb{F}}=\bar{k}$ or $\overline{\mathbb{F}}=\overline{\kappa(\mathfrak{p})}$, the group $\operatorname{Gal}(H / k)$ acts on both sides. It acts naturally on the right-hand side and the action on the left-hand side is given via the isomorphism

$$
k^{\times} \backslash \mathbb{A}_{k, f}^{\times} / \hat{\mathcal{O}}_{D}^{\times} \cong \operatorname{Gal}(H / k), \quad h \mapsto \sigma((h))
$$

given by the Artin map $\sigma$ of class field theory and the action of the idele class group given above.

The next proposition shows the compatibility of the bijections with these group actions.

Proposition 2.5 With the notation as above, the coarse moduli space map pr is compatible with these actions. More precisely, we have

$$
\operatorname{pr}_{\mathbb{F}} h \cdot(E, \iota)=\left(\operatorname{pr}_{\mathbb{F}}(E, \iota)\right)^{\sigma^{-1}(h)}
$$

Proof The compatibility over $\mathbb{C}$ is contained in the main theorem of complex multiplication [20, II, Theorem 8.2]. (Note that our normalization of the action of the class group is different from the one used by Silverman.) Precisely, we have for an elliptic curve $E=\mathbb{C} / \Lambda$ that $h . E=\mathbb{C} /(h) \Lambda$ and $j(h . E)=j^{\sigma\left(h^{-1}\right)}(E)$. Therefore, if we assume without loss of generality that $\operatorname{pr}_{\mathbb{C}}(E, \iota)=\lambda: \mathcal{O}_{H} \hookrightarrow \mathbb{C}$ is the embedding given by $j \mapsto j(E)$, then $\operatorname{pr}_{\mathbb{C}}(h .(E, \iota))=j \mapsto j(h . E)=j^{\sigma^{-1}(h)}(E)=\lambda^{\sigma^{-1}(h)}$.

We will use this to prove the statement over $\kappa(\mathfrak{p})$ for a fixed prime $\mathfrak{p}$ of $k$. Fix an isomorphism $\mathbb{C}_{\mathfrak{p}} \cong \mathbb{C}$, where $\mathbb{C}_{\mathfrak{p}}$ is the completion of an algebraic closure of $k_{\mathfrak{p}}$. Then we obtain isomorphisms

$$
C_{D}^{+}(\mathbb{C}) \cong C_{D}^{+}\left(\mathbb{C}_{\mathfrak{p}}\right) \cong C_{D}^{+}(\overline{\kappa(\mathfrak{p})})
$$

by Proposition 2.1. All of these bijections are compatible with the Serre construction. Similarly, we have bijections

$$
\operatorname{Spec} \mathcal{O}_{H}(\mathbb{C}) \cong \operatorname{Spec} \mathcal{O}_{H}\left(\mathbb{C}_{\mathfrak{p}}\right) \cong \operatorname{Spec} \mathcal{O}_{H}(\overline{\kappa(\mathfrak{p})}) .
$$

The key part is now that the diagram

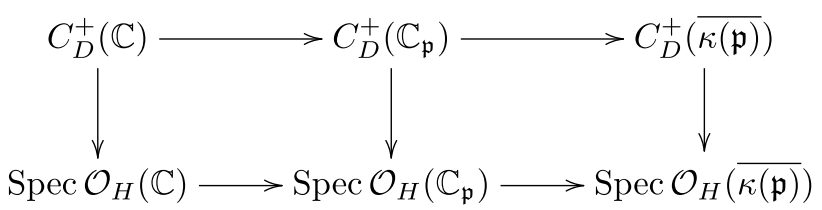

is commutative and the bijection $C_{D}^{+}(\mathbb{C}) \cong \operatorname{Spec} \mathcal{O}_{H}(\mathbb{C})$ is compatible with the actions, as stated above. The bijections in the lower row are compatible with the action of the Galois group. Consequently, the bijection $C_{D}^{+}\left(\overline{\mathbb{F}}_{p}\right) \rightarrow \operatorname{Spec} \mathcal{O}_{H}(\overline{\kappa(\mathfrak{p})})$ is compatible with the two actions, as well. 


\section{Special endomorphisms}

For $(E, \iota) \in C_{D}(S)$ we write $\mathcal{O}_{E}=\operatorname{End}_{S}(E)$ and consider the lattice $L(E, \iota)$ of special endomorphisms

$$
L(E, \iota)=\left\{x \in \mathcal{O}_{E} \mid \iota(\alpha) x=x \iota(\bar{\alpha}) \quad \text { for all } \quad \alpha \in \mathcal{O}_{D} \quad \text { and } \quad \operatorname{tr} x=0\right\}
$$

as in Definition 5.7 of [9]. It is equipped with the positive definite quadratic form $\mathrm{N}(x):=\operatorname{deg}(x)=-x^{2}$. For $S=\operatorname{Spec} \mathbb{C}$ or $S=\operatorname{Spec} \overline{\mathbb{F}}_{p}$ for a prime $p$ that is split in $k$, we have that $L(E, \iota)$ is zero.

For a non-split prime $p$ and $S=\operatorname{Spec} \overline{\mathbb{F}}_{p}, L(E, \iota)$ is a positive definite lattice of rank 2 in $\mathcal{O}_{E}$ and $(E, \iota)$ is supersingular. In this case $\mathcal{O}_{E}$ is a maximal order in the quaternion algebra $\mathbb{B}_{p}$ over $\mathbb{Q}$, which is ramified exactly at $p$ and $\infty$.

Fix a fractional ideal $\mathfrak{a} \subset k$ and let $\mu \in \mathfrak{d}_{k}^{-1} \mathfrak{a} / \mathfrak{a}$ and $m \in \mathbb{Q}_{>0}$. We write $Q(\mu)=\mathrm{N}(\mu) / \mathrm{N}(\mathfrak{a})$, which is well defined as an element of $\mathbb{Q} / \mathbb{Z}$.

The following moduli problem has been studied in $[6,9]$ and generalized in [27]. To a scheme $S$ we assign the category $\mathcal{Z}(S)$ of triples $(E, \iota, x)$, where

(i) $(E, \iota) \in C_{D}(S)$,

(ii) $x \in L(E, \iota) \mathfrak{d}_{k}^{-1} \mathfrak{a}$, such that

$$
\mathrm{N}(x)=m \mathrm{~N}(\mathfrak{a}), \quad x+\mu \in \mathcal{O}_{E} \mathfrak{a} .
$$

Here, we also wrote $\mathrm{N}(x)$ for the reduced norm in $\mathbb{B}_{p}$. If $\mathcal{Z}(S)$ is non-empty, then we have $m+\mathrm{N}(\mu) / N(\mathfrak{a}) \in \mathbb{Z}$.

Lemma 3.1 (Lemma 6.2 in [6]) The moduli problem $\mathcal{Z}$ is represented by an algebraic stack $\mathcal{Z}(m, \mathfrak{a}, \mu)$ of dimension 0 and the forgetful map $\psi: \mathcal{Z}(m, \mathfrak{a}, \mu) \rightarrow C_{D}$ defined by $(E, \iota, x) \mapsto(E, \iota)$ is finite and étale.

For $m \in \mathbb{Q}_{>0}$, we define a set of rational primes by

$$
\operatorname{Diff}(m)=\left\{p<\infty \mid(-m \mathrm{~N}(\mathfrak{a}), D)_{p}=-1\right\} .
$$

Remark 3.2 By the product formula for the Hilbert symbol [28, III.2, Theorem 3], we have

$$
\prod_{p \leq \infty}(-m \mathrm{~N}(\mathfrak{a}), D)_{p}=1 .
$$

But since $(-m \mathrm{~N}(\mathfrak{a}), D)_{\infty}=-1$, the cardinality of $\operatorname{Diff}(m)$ is odd. Moreover, if $p \in \operatorname{Diff}(m)$, then $p$ is non-split.

\section{Lemma 3.3}

(i) $\operatorname{If}|\operatorname{Diff}(m)|>1$, then $\mathcal{Z}(m, \mathfrak{a}, \mu)=\emptyset$.

(ii) If $\operatorname{Diff}(m)=\{p\}$, then $p$ is non-split in $k$ and $\mathcal{Z}(m, \mathfrak{a}, \mu)\left(\overline{\mathbb{F}}_{q}\right)=\emptyset$ for $q \neq p$.

Proof If there is an element $(E, \iota, x) \in \mathcal{Z}(m, \mathfrak{a}, \mu)$, then this shows that we have an isomorphism of quaternion algebras 


$$
\mathbb{B}_{p} \cong\left(\frac{D,-m \mathrm{~N}(\mathfrak{a})}{\mathbb{Q}}\right)
$$

However, since $\mathbb{B}_{p}$ is ramified exactly at $p$ and $\infty$, this is equivalent to

$$
(D,-m \mathrm{~N}(\mathfrak{a}))_{v}= \begin{cases}-1, & \text { if } v=p, \infty, \\ 1, & \text { otherwise. }\end{cases}
$$

This condition is equivalent to $\operatorname{Diff}(m)=\{p\}$.

In the notation of [29, Section 3], the stack $\mathcal{Z}(m, \mathfrak{a}, \mu)$ defines a 0-cycle $\psi_{*}[\mathcal{Z}(m, \mathfrak{a}, \mu)]$ on $C_{D}$ since the forgetful map is proper.

Moreover, note that the map pr: $C_{D} \rightarrow \operatorname{Spec} \mathcal{O}_{H}$ is also proper by Lemma 2.2. Therefore, we can consider the proper pushforward $\operatorname{pr}_{*}[\mathcal{Z}(m, \mathfrak{a}, \mu)]$ to $\operatorname{Spec} \mathcal{O}_{H}$. In our case

$$
\operatorname{pr}_{*}[\mathcal{Z}(m, \mathfrak{a}, \mu)]=\frac{1}{w_{k}}[\operatorname{pr}(\mathcal{Z}(m, \mathfrak{a}, \mu))]
$$

because the automorphism group of a general geometric point of $C_{D}$ is $\mathcal{O}_{D}^{\times}[9]$.

By abuse of notation, we also denote by $\mathcal{Z}(m, \mathfrak{a}, \mu)$ the corresponding divisor on the coarse moduli scheme and simply write

$$
\mathcal{Z}(m, \mathfrak{a}, \mu)=\sum_{\mathfrak{P} \subset \mathcal{O}_{H}} \mathcal{Z}(m, \mathfrak{a}, \mu)_{\mathfrak{P}} \mathfrak{P} .
$$

If there is no confusion possible, we simply write $\mathcal{Z}(m)$ or $\mathcal{Z}(m)_{\mathfrak{P}}$. Note that the multiplicities above are the same for the pushforward from $C_{D}^{+}$and from $C_{D}$.

In what follows, we will find formulas for the multiplicities $\mathcal{Z}(m, \mathfrak{a}, \mu)_{\mathfrak{P}}$. From now on, fix a prime $p$ that is non-split in $k$ and assume that $m \equiv-Q(\mu) \bmod \mathbb{Z}$. Let $p_{0} \in \mathbb{Z}$ be a prime with $p_{0} \nmid 2 p D$ such that if $p$ is inert in $k$, we have

$$
\left(D,-p p_{0}\right)_{v}= \begin{cases}-1, & v=p, \infty \\ 1, & \text { otherwise }\end{cases}
$$

and if $p$ is ramified in $k$, we have

$$
\left(D,-p_{0}\right)_{v}= \begin{cases}-1, & v=p, \infty \\ 1, & \text { otherwise }\end{cases}
$$

With this choice, put

$$
\kappa_{p}= \begin{cases}p p_{0}, & \text { if } p \text { is inert in } k \\ p_{0}, & \text { if } p \text { is ramified in } k\end{cases}
$$

and let $\mathfrak{p}_{0}$ be a fixed prime ideal of $\mathcal{O}_{D}$ lying above $p_{0}$. Here, $(\cdot, \cdot)_{v}$ denotes the $v$-adic Hilbert symbol. The existence of such a prime $p_{0}$ follows essentially from Dirichlet's theorem, see also [28, III, Theorem 4]. Note that the genus of $\left[\mathfrak{p}_{0}\right]$ is well defined since the symbols $(D, \cdot)_{\nu}$ form a basis for the genus characters.

We can write $\mathbb{B}_{p}=k \oplus k y_{0}$, where $y_{0}^{2}=\kappa_{p}$. Here, the decomposition is orthogonal with respect to the bilinear form corresponding to the reduced norm of $\mathbb{B}_{p}$. We write $[\gamma, \delta]$ for the element $\gamma+\delta y_{0} \in \mathbb{B}_{p}$. 
Proposition 3.4 Let $p$ be a prime that is non-split in $k$ and let $(E, \iota) \in C_{D}\left(\overline{\mathbb{F}}_{p}\right)$. Then $L(E, \iota)$ is a projective $\mathcal{O}_{D}$-module of rank 1 and there is a fractional ideal $\mathfrak{b} \subset k$, such that

$$
L(E, \iota) \cong \mathfrak{b}^{-1} \mathfrak{p}_{0}^{-1} y_{0} .
$$

Here $y_{0} \in \mathcal{O}_{E}$ with $\mathrm{N}\left(y_{0}\right)=\kappa_{p}$. Moreover, if $\in \mathbb{A}_{k, f}^{\times}$, then

$$
L(h .(E, \iota))=(h) \overline{(h)}^{-1} L(E, \iota) .
$$

Proof The first statement is Proposition 5.13 in [9]. The second follows from the action of the ideles on maximal orders in the quaternion algebra $\mathbb{B}_{p}$, as described in detail in Section 5 of [9].

Recall that there are two actions of $\mathbb{A}_{k, f}^{\times}$on ideals of $k$. One is given by multiplication by the ideal $(h)$ corresponding to the idele $h$ and the other one is given by the action of $\mathbb{A}_{k, f}^{\times} \cong T\left(\mathbb{A}_{f}\right)$, where $T=\operatorname{GSpin}_{U}$ for the quadratic space $U=k$ with quadratic form given by the norm on $k$. For details, we refer to Section 2.2 of [30]. To avoid confusion, we will denote the action of $h$ as an element of $T\left(\mathbb{A}_{f}\right)$ by $h \cdot \mathfrak{a}=(h)(\bar{h})^{-1} \mathfrak{a}$ for any fractional ideal $\mathfrak{a} \subset k$.

Proposition 3.5 Let $h \in \mathbb{A}_{k, f}^{\times}$and write $\sigma=\sigma(h)$ for the element of $\mathrm{Gal}(H / k)$ under the Artin map. Then we have

$$
\mathcal{Z}(m, \mathfrak{a}, \mu)_{\mathfrak{P}^{\sigma}}=\mathcal{Z}\left(m, h^{-1} \cdot \mathfrak{a}, h^{-1} \cdot \mu\right)_{\mathfrak{P}} .
$$

Proof This follows from Propositions 2.5 and 3.4.

Lemma 3.6 Let $\left(E_{0}, \iota_{0}\right) \in C_{D}\left(\overline{\mathbb{F}}_{p}\right)$ such that $\left[L\left(E_{0}, \iota_{0}\right)\right]^{-1}=\left[\mathfrak{p}_{0}\right]$ where $\left[L\left(E_{0}, \iota_{0}\right)\right]$ denotes the class of the rank one $\mathcal{O}_{D}$-module in $\operatorname{Pic}\left(\mathcal{O}_{D}\right)$. Then the maximal order $\operatorname{End}\left(E_{0}\right)$ of $\mathbb{B}_{p}$ can be described in the following way:

If $p$ is inert in $k$, let $\mathfrak{c}_{0}=\mathfrak{p}_{0} \mathfrak{d}_{k}$. If $p$ is ramified and $\mathfrak{p} \subset \mathcal{O}_{D}$ is the prime above $p$, let $\mathfrak{c}_{0}=\mathfrak{p}_{0} \mathfrak{p}^{-1} \mathfrak{d}_{k}$.

There exists a generator $\lambda_{0}$ of $\mathfrak{d}_{k}^{-1} \mathfrak{c}_{0} / \mathfrak{c}_{0}$ with

$$
\mathrm{N}\left(\lambda_{0}\right) \equiv-\kappa_{p} \bmod \mathrm{N}\left(\mathfrak{c}_{0}\right),
$$

such that

$$
\operatorname{End}\left(E_{0}, \iota_{0}\right)=\mathcal{O}_{\mathfrak{c}_{0}, \lambda_{0}, \mathbb{B}_{p}},
$$

where

$$
\mathcal{O}_{\mathfrak{c}_{0}, \lambda_{0}, \mathbb{B}_{p}}=\left\{[\gamma, \delta] \mid \gamma \in \mathfrak{d}_{k}^{-1}, \delta \in \mathfrak{c}_{0}^{-1}, \gamma+\lambda \delta \in \mathcal{O}_{D}\right\}
$$

is a maximal order in $\mathbb{B}_{p}$.

Moreover, if $(E, \iota)=h .\left(E_{0}, \iota_{0}\right)$, we have

$$
\operatorname{End}(E, \iota)=\mathcal{O}_{h . c_{0}, h . \lambda, \mathbb{B}_{p}} .
$$


Proof See Lemma 3.3 and Lemma 7.1 of [27]. The result has also been described by Dorman $[4,31]$.

Remark 3.7 There are $2^{t}$ possible choices for generator of $\mathfrak{d}_{k}^{-1} \mathfrak{c}_{0} / \mathfrak{c}_{0}$, with the required norm, where $t$ is the number of prime divisors of $D$. However, there are only $2^{t-1}$ inequivalent ones. Here, we consider the orders to be equivalent if they are conjugate by an element of $k^{\times}$. Indeed, $\lambda$ and $-\lambda$ yield such equivalent conjugate orders.

However, not knowing the specific $\lambda$ that corresponds to the chosen point $\left(E_{0}, \iota_{0}\right)$ results in an ambiguity in Proposition 3.8 below, cf. [4]. We will resolve this issue later on by taking the relative norm to the fixed field of all elements of order dividing 2 in the Galois group $\mathrm{Gal}(H / k)$.

In the most general case that we consider, the multiplicities involve representation numbers with additional congruences that we will define now. For a fractional ideal $\mathfrak{a}$ of $k$, we let $\mathfrak{c}_{\mathfrak{a}}=\mathfrak{a} \overline{\mathfrak{a}}^{-1} \mathfrak{c}_{0}$. Moreover, we let $\lambda_{\mathfrak{a}}=a . \lambda_{0} \in \mathfrak{d}_{k}^{-1} \mathfrak{c}_{\mathfrak{a}} / \mathfrak{c}_{\mathfrak{a}}$, where $a \in \mathbb{A}_{k, f}^{\times}$is an idele determining $\mathfrak{a}$ and $\lambda_{0}$ is given in Lemma 3.6. Note that $a$ is only unique up to an element of $\hat{\mathcal{O}}_{D}^{\times}$but $\lambda_{\mathfrak{a}}$ is a well defined element of $\mathfrak{d}_{k}^{-1} \mathfrak{c}_{\mathfrak{a}} / \mathfrak{c}_{\mathfrak{a}}$ since $\hat{\mathcal{O}}_{D}^{\times}$acts trivially on $\mathfrak{d}_{k}^{-1} \mathfrak{c}_{\mathfrak{a}} / \mathfrak{c}_{\mathfrak{a}}$.

For $n \in \mathbb{Q}_{>0}$ and $\mu \in \mathfrak{d}_{k}^{-1} \mathfrak{a} / \mathfrak{a}$, we let

$$
\rho_{0}(n, \mathfrak{a}, \mu)=\#\left\{x \in \mathfrak{c}_{\mathfrak{a}}^{-1} \mathfrak{a}=\mathfrak{c}_{0}^{-1} \overline{\mathfrak{a}} \mid \mathrm{N}(x)=n, \lambda_{\mathfrak{a}} x+\mu \in \mathfrak{a}\right\} .
$$

We need to define one more quantity to describe the multiplicities $\mathcal{Z}(m, \mathfrak{a}, \mu)_{\mathfrak{P}}$.

Let $p$ be a prime which is non-split in $k$ and define

$$
v_{p}(m)= \begin{cases}\frac{1}{2}\left(\operatorname{ord}_{p}(m)+1\right), & \text { if } p \text { is inert in } k \\ \operatorname{ord}_{p}(m|D|), & \text { if } p \text { is ramified in } k .\end{cases}
$$

Note that the prime ideals $\mathfrak{P} \mid p$ of $H$ correspond to the irreducible components of Spec $\mathcal{O}_{H}\left(\overline{\mathbb{F}}_{p}\right)$. We let $\mathfrak{P}_{0}$ be the prime ideal such that $\operatorname{pr}_{\mathbb{F}_{p}}\left(E_{0}, \iota_{0}\right)$ with $\left(E_{0}, \iota_{0}\right)$ as in Lemma 3.6 lies in the irreducible component corresponding to $\mathfrak{P}_{0}$.

Proposition 3.8 Suppose that $\operatorname{Diff}(m)=\{p\}$.

(i) We have $\mathcal{Z}(m, \mathfrak{a}, \mu)_{\mathfrak{P}}=0$ unless $m+Q(\mu) \in \mathbb{Z}$.

(ii) For $m+Q(\mu) \in \mathbb{Z}$, we have

$$
\mathcal{Z}(m, \mathfrak{a}, \mu)_{\mathfrak{P}_{0}}=\frac{v_{p}(m)}{w_{k}} \rho_{0}\left(\frac{m}{\kappa_{p}} \mathrm{~N}(\mathfrak{a}), \mathfrak{a}, \mu\right) .
$$

Proof First note that our cycles correspond to those studied in [27] which are generalizations of those in [12]. The cycle $\mathcal{Z}(m, \mathfrak{a}, \mu)$ corresponds to $\mathcal{Z}\left(m|D| ; \mathfrak{d}_{k} \mathfrak{a}^{-1}, \lambda^{\prime}, \lambda^{\prime} \mu\right)$ for a generator $\lambda^{\prime} \in \mathfrak{a}^{-1} / \mathfrak{a}^{-1} \mathfrak{d}_{k}$.

The push-forward $\operatorname{pr}_{*}[\mathcal{Z}(m)]$ is given by a formal sum

$$
\sum_{\mathfrak{P} \subset \mathcal{O}_{H}} n_{\mathfrak{P}} \text {. }
$$

We will now determine the multiplicities. Fix a rational prime $p$ and a prime ideal $\mathfrak{P} \subset \mathcal{O}_{H}$ over $p$. Moreover, fix any geometric point $\xi=\left(E_{0}, \iota_{0}\right) \in C_{D}(\overline{\kappa(\mathfrak{P})})$. Using 
Propositon 4.1 in [27], we see that the length $\lg \hat{\mathcal{O}}_{\mathcal{Z}(m), \xi}$ of the completed local ring is given by

$$
\lg \hat{\mathcal{O}}_{\mathcal{Z}(m), \xi}=v_{p}(m)
$$

Note that in the notation of [27], we have $\partial=\mathfrak{d}_{k}=\partial_{\lambda}$ and $\Delta=D$. Moreover, $\operatorname{ord}_{p}(m)=\operatorname{ord}_{p}(m|D|)$ for $p \nmid D$. Therefore,

$$
n_{\mathfrak{P}}=\frac{v_{p}(m)}{w_{k}} \cdot \#\left\{x \in L(\xi) \mid(\xi, x) \in \mathcal{Z}(m)\left(\overline{\mathbb{F}}_{p}\right)\right\} .
$$

Thus, what is left is to count the number of endomorphisms $x$, such that $\left(E_{0}, \iota_{0}, x\right)=(\xi, x) \in \mathcal{Z}(m)(\overline{\kappa(\mathfrak{P})})$. That is, we need to count the number of $x \in L\left(E_{0}, \iota_{0}\right) \mathfrak{d}_{k}^{-1} \mathfrak{a}$, such that

$$
\mathrm{N}(x)=m \mathrm{~N}(\mathfrak{a}), \quad x+\mu \in \mathcal{O}_{E_{0}} \mathfrak{a} .
$$

The endomorphism ring $\mathcal{O}_{E_{0}}=\operatorname{End}\left(E_{0}\right)$ is a maximal order contained in the quaternion algebra $\mathbb{B}_{p}=k \oplus k y_{0}$, where $y_{0}^{2}=\kappa_{p}$. By Lemma 3.6, we have

$$
\mathcal{O}_{E_{0}}=\mathcal{O}_{\mathfrak{c}_{0}, \lambda_{0}, \mathbb{B}}=\left\{[\gamma, \delta] \mid \gamma \in \mathfrak{d}_{k}^{-1}, \delta \in \mathfrak{c}_{0}^{-1}, \gamma+\lambda_{0} \delta \in \mathcal{O}_{D}\right\}
$$

This implies that

$$
L\left(E_{0}, \iota_{0}\right)=\mathcal{O}_{0} \cap k y_{0}=\mathfrak{c}_{0}^{-1} \mathfrak{d}_{k} y_{0} .
$$

An element $x \in L\left(E_{0}, \iota_{0}\right) \mathfrak{d}_{k}^{-1} \mathfrak{a}$ is, therefore, of the form $x=\alpha y_{0}$ for $\alpha \in \mathfrak{c}_{0}^{-1} \overline{\mathfrak{a}}$. By Proposition 7.1 of [27], we have that

$$
\mathfrak{a} \mathcal{O}_{\mathfrak{c}_{\mathfrak{a}}, \lambda_{\mathfrak{a}}, \mathbb{B}_{p}}=a \mathcal{O}_{\mathfrak{c}_{\mathfrak{a}}, \lambda_{\mathfrak{a}}, \mathbb{B}_{p}}=\mathcal{O}_{\mathfrak{c}_{0}, \lambda_{0}, \mathbb{B}_{p}} a=\mathcal{O}_{\mathfrak{c}_{0}, \lambda_{0}, \mathbb{B}_{p}} \mathfrak{a}
$$

where $a \in \mathbb{A}_{k, f}^{\times}$is an idele determining $\mathfrak{a}$. Note that this does not depend on the choice of such an $a$ because the order is invariant under the action of $\hat{\mathcal{O}}_{D}^{\times}$.

Consequently, the condition $\mu+\alpha y_{0} \in \mathcal{O}_{E_{0}} \mathfrak{a}$ is equivalent to $\mu \in \mathfrak{d}_{k}^{-1} \mathfrak{a}$ and $\alpha \in \mathfrak{c}_{0}^{-1} \overline{\mathfrak{a}}$ such that $\mu+\lambda_{\mathfrak{a}} \alpha \in \mathfrak{a}$. The norm of $\alpha$ is required to be $\mathrm{N}(\alpha)=\left(m / \kappa_{p}\right) \mathrm{N}(\mathfrak{a})$. This yields the representation number $\rho_{0}\left(\left(m / \kappa_{p}\right) \mathrm{N}(\mathfrak{a}), \mathfrak{a}, \mu\right)$ and ends the proof.

We can avoid the ambiguity in the formulas above by taking the quotient $\mathrm{Cl}_{\mathrm{k}} / \mathrm{Cl}_{\mathrm{k}}[2]$ by the subgroup $\mathrm{Cl}_{\mathrm{k}}$ [2] of elements of order dividing 2. This corresponds to calculating the valuation at primes $\ell \subset \mathcal{O}_{L}$, where $L \subset H$ is the subfield fixed by all elements of order 2 in $\operatorname{Gal}(H / k)$. We obtain a 0 -cycle on $\operatorname{Spec} \mathcal{O}_{L}$ via the projection $\operatorname{Spec} \mathcal{O}_{H} \rightarrow \operatorname{Spec} \mathcal{O}_{L}$.

For an ideal class $[c] \in \mathrm{Cl}_{\mathrm{k}}$ and a positive integer $n$ we define the representation number

$$
\rho(n,[\mathfrak{c}])=\left|\left\{\mathfrak{b} \subset \mathcal{O}_{D} \mid \mathrm{N}(\mathfrak{b})=n, \mathfrak{b} \in[\mathfrak{c}]\right\}\right| .
$$

We obtain the following, simpler result:

Proposition 3.9 Let $L \subset H$ be the fixed field of $\operatorname{Gal}(H / k)[2]$, where $H$ is the Hilbert class field of $k$. Let $[\mathrm{c}] \in \mathrm{Cl}_{\mathrm{k}}$ be an ideal class and let $\sigma$ correspond to $[\mathfrak{c}]$ under the Artin map. Moreover, let $\mathfrak{f} \subset \mathcal{O}_{L}$ be the prime ideal below $\mathfrak{P}_{0}$. We have for $m+Q(\mu) \in \mathbb{Z}$ that 


$$
\mathcal{Z}(m, \mathfrak{a}, \mu)_{\mathfrak{f}^{\sigma}}=2^{o(m)-1} v_{p}(m) \rho\left(m|D| / p,[\mathfrak{c}]^{-2}\left[\mathfrak{c}_{0} \mathfrak{a}\right]\right),
$$

where $v_{p}(m)$ is given before Proposition 3.8 and $o(m)$ is the number of primes $p \mid D$ such that $\operatorname{ord}_{p}(m|D|)>0$.

Proof It is enough to consider the case $[\mathfrak{c}]=\left[\mathcal{O}_{D}\right]$, that is, to determine the multiplicity for the prime $\mathfrak{f}$. The general formula follows by the action of the Galois group given in Proposition 3.5. We need to calculate the sum

$$
f \sum_{\mathfrak{P} \mid \mathfrak{f}} \mathcal{Z}(m, \mathfrak{a}, \mu)_{\mathfrak{P}}=\sum_{\tau \in \operatorname{Gal}(H / L)} \mathcal{Z}(m, \mathfrak{a}, \mu)_{\mathfrak{P}_{0}^{\tau}}
$$

where $f=2$ if $p$ is ramified in $k$ and $D$ is not a prime and $f=1$, otherwise. (This is the ramification degree of $\mathfrak{P} \mid \mathfrak{f}$.) According to Propositions 3.5 and 3.8, this is equal to

$$
\sum_{\tau \in \operatorname{Gal}(H / L)} \mathcal{Z}(m, \mathfrak{a}, \mu)_{\mathfrak{P}_{0}^{\tau}}=\frac{1}{w_{k}} v_{p}(m) \sum_{\substack{h \in \mathcal{C} \\ h^{2}=1}} \rho_{0}\left(\frac{m}{\kappa_{p}} \mathrm{~N}(\mathfrak{a}), h^{-1} \cdot \mathfrak{a}, h^{-1} \cdot \mu\right),
$$

where $\mathcal{C}=k^{\times} \backslash \mathbb{A}_{k, f}^{\times} / \hat{\mathcal{O}}_{k}^{\times} \cong \mathrm{Cl}_{\mathrm{k}}$ acts as $\mathrm{GSpin}_{U}\left(\mathbb{A}_{f}\right)$ for $U=k$ as described above. The elements of order less or equal to 2 in the class group $\mathrm{Cl}_{\mathrm{k}}$ correspond to the prime divisors of $D$. If $h^{2}=1$, then $h \cdot \mathfrak{a}=\mathfrak{a}$ because $\mathfrak{p} / \overline{\mathfrak{p}}=\mathcal{O}_{D}$ for prime divisors of $\mathfrak{d}_{k}$. As $h$ ranges over $\mathcal{C}$ [2], h. $\mu$ runs through a set of representatives of all $\beta \in \mathfrak{d}_{k}^{-1} \mathfrak{a} / \mathfrak{a}$ with $\mathrm{N}(\beta) \equiv \mathrm{N}(\mu) \bmod \mathrm{N}(\mathfrak{a})$ modulo the action of \pm 1 . Each of these $\beta$ is counted with multiplicity

$$
\begin{cases}2^{o(m)-1}, & \text { if } o(m) \geq 1 \\ 1, & \text { otherwise. }\end{cases}
$$

Finally, if $\alpha \in \mathfrak{c}_{0}^{-1} \overline{\mathfrak{a}}$ with $\mathrm{N}(\alpha)=\left(m / \kappa_{p}\right) \mathrm{N}(\mathfrak{a})$, then $\tilde{\mathfrak{a}}=\alpha \mathfrak{c}_{0} \overline{\mathfrak{a}}^{-1} \subset \mathcal{O}_{D}$ is an integral ideal with

$$
\mathrm{N}(\tilde{\mathfrak{a}})=\frac{m}{\kappa_{p}} \cdot \frac{\kappa_{p}|D|}{p}=\frac{m|D|}{p}
$$

which lies in the class $[\tilde{\mathfrak{a}}]=\left[\mathfrak{c}_{0} \mathfrak{a}\right]$. In this correspondence $\alpha \mapsto \tilde{\mathfrak{a}}$, each ideal occurs with multiplicity

$$
\frac{w_{k}}{2} \cdot \begin{cases}2 & \text { if } o(m) \geq 1 \\ 1 & \text { otherwise }\end{cases}
$$

because $-\mu$ is in the set $\left\{h \cdot \mu \mid h^{2}=1\right\}$ if and only if $o(m)=o(\mu) \geq 1$.

Proposition 3.10 Let $D=-l$ for a prime $l \equiv 3 \bmod 4$. Let $m \in \mathbb{Q}$ and assume that $\operatorname{Diff}(m)=\{p\}$ and $m+Q(\mu) \in \mathbb{Z}$. Fix an embedding of $H=k(j)$ into $\mathbb{C}$.

There is a unique prime ideal $\mathfrak{P} \mid p$ of $H$ fixed by complex conjugation, $\overline{\mathfrak{P}}=\mathfrak{P}$ and we have 


$$
\mathcal{Z}(m, \mathfrak{a}, \mu)_{\mathfrak{P}}=2^{o(m)-1} v_{p}(m) \rho(m|D| / p,[\mathfrak{a}]) .
$$

Proof First note that the class number of $h_{k}$ is odd [32]. Since $p$ is non-split in $k$, the unique prime $\mathfrak{p} \subset \mathcal{O}_{D}$ above $p$ splits completely in $H$. Therefore, the number of primes of $\mathcal{O}_{H}$ above $p$ is odd and there is at least one prime fixed by complex conjugation. Let $\mathfrak{P}$ be such a prime. Let $\tau$ denote complex conjugation $x \mapsto \bar{x}$. Since $\sigma \circ \tau=\tau \circ \sigma^{-1}$ for all $\sigma \in \operatorname{Gal}(H / k)$, we have

$$
\mathfrak{P}^{\sigma\left(\mathfrak{b}^{-1}\right)}=\overline{\mathfrak{P}^{\sigma(\mathfrak{b})}}
$$

for every $\mathfrak{b}$. Suppose that $\mathfrak{Q}$ is another prime above $\mathfrak{p}$ with $\mathfrak{Q}=\overline{\mathfrak{Q}}$ and $\mathfrak{Q}=\mathfrak{P}^{\sigma(\mathfrak{b})}$. Then it is easy to see that $\mathfrak{P}^{\sigma\left(\mathfrak{b}^{2}\right)}=\mathfrak{P}$. Thus, since $\operatorname{Gal}(H / k)$ acts transitively on the set of primes above $\mathfrak{p}$ and $\mathfrak{p}$ is totally split in $H$, we have that $\sigma\left(\mathfrak{b}^{2}\right)$ is the identity and thus $[\mathfrak{b}]^{2}=\left[\mathcal{O}_{D}\right]$. Since $h_{k}$ is odd, this implies that $[\mathfrak{b}]=\left[\mathcal{O}_{D}\right]$ and, therefore, $\mathfrak{P}$ is the only prime fixed by complex conjugation.

Let $W$ be the completion of the maximal unramified extension of $\mathcal{O}_{H, \mathfrak{P}}$ (here, $\mathcal{O}_{H, \mathfrak{P}}$ is the completion of $\mathcal{O}_{H}$ with respect to $\left.\mathfrak{P}\right)$. Fix an algebraic closure $\overline{\kappa(\mathfrak{p})}$ of the residue field $\kappa(\mathfrak{p})=\mathcal{O}_{D} / \mathfrak{p}$. The ring $W$ is a complete discrete valuation ring with maximal ideal $\pi$ and its residue field $W / \pi$ is algebraically closed and, therefore, isomorphic to $\overline{\kappa(\mathfrak{P})} \cong \overline{\kappa(\mathfrak{p})}$ (see Corollary 1 of Chapter II in [33]). Recall the diagram in the proof of Proposition 2.5. We can consider a similar diagram with $W$ in place of $\mathbb{C}_{\mathfrak{p}}$. The bijection $C_{D}^{+}(\mathbb{C}) \rightarrow C_{D}^{+}(W)$ is obtained by mapping a CM elliptic curve $(E, \iota) \in \mathbb{C}_{D}^{+}(\mathbb{C})$ to an elliptic curve $(\tilde{E}, \iota)$ over $W$ with $j$-invariant $j(\tilde{E})=j(E)$. Such an elliptic curve with good reduction exists by the theorem of Serre and Tate $[3,34]$ and is unique up to $W$-isomorphism. That all the maps involved are bijections is a consequence of the canonical lifting theorem [22, 23].

Now let $E$ be an elliptic curve over $W$ with $j$-invariant $j(E)=j\left(E_{\mathcal{O}_{D}}\right)$. Using the description above, we see that the reduction of $E$ maps to the homomorphism $\mathcal{O}_{H} \rightarrow \overline{\kappa(\mathfrak{p})}$ such that the image of $j\left(E_{\mathcal{O}_{D}}\right)$ is contained in $\mathbb{F}_{p}$, which corresponds to $\mathfrak{P}$.

As in Lemma 3.5 of [3], we have that $\operatorname{End}_{W / \pi}(E)$ is isomorphic to $\mathcal{O}_{\mathcal{O}_{D}, \lambda, \mathbb{B}_{p}}$, where $\lambda$ is any of the two possible $\lambda \in \mathcal{O}_{D} / \mathfrak{d}_{k}$ with $\mathrm{N}(\lambda) \equiv-p \bmod |D|$. Therefore, $\mathfrak{P}=\mathfrak{P}_{0}^{\sigma}$, where $\sigma=\sigma(\mathfrak{b})$ with $[\mathfrak{b}]^{2}=\left[\mathfrak{c}_{0}\right]=\left[\mathfrak{p}_{0}\right]$.

Thus,

$$
\mathcal{Z}(m, \mathfrak{a}, \mu)_{\mathfrak{P}}=\mathcal{Z}(m, \mathfrak{a}, \mu)_{\mathfrak{P}_{0}^{\sigma}}=2^{o(m)-1} v_{p}(m) \rho(m|D| / p,[\mathfrak{a}])
$$

by means of Proposition 3.9.

We can now also give a formula for the Arakelov degree $\widehat{\operatorname{deg}} \mathcal{Z}(m, \mathfrak{a}, \mu)$.

Following [27], we define

$$
\begin{aligned}
\widehat{\operatorname{deg} \mathcal{Z}(m, \mathfrak{a}, \mu)} & =\sum_{p} \log p \sum_{x \in \mathcal{Z}(m, \mathfrak{a}, \mu)\left(\overline{\mathbb{F}}_{p}\right)} \frac{1}{\left|\operatorname{Aut}_{C_{D}}(\varphi(x))\right|} \lg (x) \\
& =\frac{1}{w_{k}} \sum_{p} \log (p) \sum_{x \in \mathcal{Z}(m, \mathfrak{a}, \mu)\left(\overline{\mathbb{F}}_{p}\right)} \lg (x)
\end{aligned}
$$


and the sum runs over all rational primes. Here, we define

$$
\lg (x)=\text { length of } \mathcal{O}_{\mathcal{Z}(m, \mathfrak{a}, \mu), x}=\text { length of } \hat{\mathcal{O}}_{\mathcal{Z}(m, \mathfrak{a}, \mu), x} .
$$

This definition can also be expressed as $\widehat{\operatorname{deg}} \mathcal{Z}(m, \mathfrak{a}, \mu)=\widehat{\operatorname{deg}} \operatorname{pr}_{*}[\mathcal{Z}(m, \mathfrak{a}, \mu)]$, where the latter is the usual Arakelov degree of an arithmetic divisor on the arithmetic curve given by $\operatorname{Spec} \mathcal{O}_{H}$. We use Proposition 3.9 to calculate this degree by using that the degree map is compatible with pushforward. We have proved the following result, which is one of the results of $[9,27]$ :

Corollary 3.11 Assume that $m+Q(\mu) \in \mathbb{Z}$ and $\operatorname{Diff}(m)=\{p\}$. Then we have

$$
\widehat{\operatorname{deg}} \mathcal{Z}(m, \mathfrak{a}, \mu)=2^{o(m)-1}\left(\operatorname{ord}_{p}(m)+1\right) \rho\left(m|D| / p,\left[\left[\mathfrak{c}_{0} \mathfrak{a}\right]\right]\right) \log (p),
$$

where $\rho(n,[[\mathfrak{b}]])$ is the number of integral ideals of $\mathcal{O}_{D}$ of norm $n$ in the genus of $\mathfrak{b}$.

\section{An integral model for the modular curve}

We recall some of the properties of the integral model for the modular curve $Y_{0}(N)$ and its compactification $X_{0}(N)$. These models have been intensively studied by Deligne and Rapoport [35], Katz and Mazur [18]. We refer to these references and [36] for details.

The stack $\mathcal{Y}_{0}(N)\left(\mathcal{X}_{0}(N)\right)$ over $\mathbb{Z}$ represents the moduli problem that assigns to any base scheme $S$ the cyclic isogenies of degree $N$ of (generalized) elliptic curves $\pi: E \rightarrow E^{\prime}$ over $S$ such that ker $\pi$ meets every irreducible component of each geometric fiber. On complex points, we have $\mathcal{Y}_{0}(N)(\mathbb{C})=Y_{0}(N)(\mathbb{C})$ and $\mathcal{X}_{0}(N)(\mathbb{C})=X_{0}(N)(\mathbb{C})$.

Here, the condition that $A=\operatorname{ker} \pi$ is cyclic of degree $N$ means that locally on $S$ there is a point $P$ such that

$$
A=\sum_{a=1}^{N}[a P]
$$

as a Cartier divisor on $E$. This becomes the usual condition that $A$ is locally isomorphic to $\mathbb{Z} / N \mathbb{Z}$, when $N$ is invertible in $S$. We will always assume that $N$ is square-free. In this case the condition means that $A$ is locally free of rank $N$.

The cusps correspond to certain degenerated elliptic curves [35]. We will not give a precise definition of these as we will mostly work on the substack $\mathcal{Y}_{0}(N)$.

Theorem 4.1 (Theorems 1.2.1 and 3.2.7 of [37]) Let $N$ be square-free. Then the stack $\mathcal{X}_{0}(N)$ is a proper flat Deligne-Mumford stack over $\mathbb{Z}$. It is regular and has geometrically connected fibers of pure dimension one. Moreover, the stack $\mathcal{X}_{0}(N)$ is smooth over $\mathbb{Z}[1 / N]$.

\subsection{Integral extensions of Heegner divisors}

The following moduli problem describes a natural extension of the divisor $Z(d, r)$ defined in the introduction to the stack $\mathcal{X}_{0}(N)$. We follow [6, Section 7.3].

Definition 4.2 Let $d \in \mathbb{Z}_{<0}$ and $r \in \mathbb{Z}$ such that $d \equiv r^{2} \bmod 4 N$. The integer $d$ is a negative discriminant and we denote by $\mathcal{O}_{d}$ the order of $k=\mathbb{Q}(\sqrt{d})$ of discriminant $d$. The 
ideal $\mathfrak{n}=\left(N, \frac{r+\sqrt{d}}{2}\right)$ has norm $N$. We define $\mathcal{Z}(d, r)$ to be the Deligne-Mumford stack representing the moduli problem which assigns to a base scheme $S$ over $\mathbb{Z}$ the set of pairs $\left(\pi: E \rightarrow E^{\prime}, \iota\right)$, such that

(i) $\pi: E \rightarrow E^{\prime}$ is a cyclic degree $N$ isogeny of elliptic curves $E$ and $E^{\prime}$ over $S$,

(ii) $\iota: \mathcal{O}_{d} \hookrightarrow \operatorname{End}(\pi)=\left\{\alpha \in \operatorname{End}(E): \pi \alpha \pi^{-1} \in \operatorname{End}\left(E^{\prime}\right)\right\}$ is an $\mathcal{O}_{d \text {-action on } \pi}$ such that $\iota(\mathfrak{n}) \operatorname{ker} \pi=0$.

There is a natural morphism

$$
\mathcal{Z}(d, r) \longrightarrow \mathcal{X}_{0}(N)
$$

given by the forgetful map $\left(\pi: E \rightarrow E^{\prime}, \iota\right) \mapsto \pi: E \rightarrow E^{\prime}$. Note that $\mathcal{Z}(d, r)$ does not intersect the boundary $\mathcal{X}_{0}(N) \backslash \mathcal{Y}_{0}(N)$ [26]. We recall the following two facts from [6]:

Lemma 4.3 The forgetful map $\mathcal{Z}(d, r) \longrightarrow \mathcal{X}_{0}(N)$ is finite and étale. The stack $\mathcal{Z}(d, r)$ defines a horizontal divisor on $\mathcal{X}_{0}(N)$.

Lemma 4.4 As divisors in the complex fiber, we have

$$
\mathcal{Z}(d, r)(\mathbb{C})=Z(d, r)
$$

The divisor $\mathcal{Z}(d, r)$ is in fact the flat closure of $Z(d, r)$.

We briefly mention the relation to the Heegner points as defined by Birch [38], Gross [39] and Gross and Zagier [36]. A Heegner point on $X_{0}(N)(\mathbb{C})$ is described by the data $(\mathcal{O}, \mathfrak{n},[\mathfrak{a}])$, where $\mathcal{O} \subset k$ is an order, $\mathfrak{n} \subset \mathcal{O}$ is a proper $\mathcal{O}$-ideal with quotient $\mathcal{O} / \mathfrak{n}$ cyclic of order $N$ and $[\mathfrak{a}]$ is the class of some invertible $\mathcal{O}$-module $\mathfrak{a}$ in $\operatorname{Pic}(\mathcal{O})$. The Heegner point corresponding to this data is given by the diagram

$$
\mathbb{C} / \mathfrak{a} \rightarrow \mathbb{C} / \mathfrak{a n}^{-1} .
$$

If we choose an oriented basis $\left(\omega_{1}, \omega_{2}\right)$ of $\mathfrak{a}$, such that $\mathfrak{a n}^{-1}=\left(\omega_{1}, \omega_{2} / N\right)$, then the point in $X_{0}(N)(\mathbb{C}) \cong \Gamma_{0}(N) \backslash \mathbb{H}$ is given by the orbit of $\tau=\omega_{1} / \omega_{2}[39]$.

\section{$7 \mathrm{CM}$ values of modular functions}

The starting point for our study of CM values of modular functions with zeros and poles supported on Heegner divisors is the following Lemma:

Lemma 5.1 [6, Lemma 7.10] Let $D$ be a negative fundamental discriminant and assume that $D \equiv 1 \bmod 4$. Let $r \in \mathbb{Z}$ such that $D \equiv r^{2} \bmod 4 N$. There is an isomorphism of stacks

$$
\mathbf{j}_{D}: C_{D} \rightarrow \mathcal{Z}(D, r), \quad(E, \iota) \mapsto(\pi: E \rightarrow E / E[\mathfrak{n}], \iota) .
$$

Here, we denote by $E[\mathfrak{n}]$ the kernel of multiplication by elements in $\mathfrak{n}$. Combining the map $\mathbf{j}_{D}$ with the forgetful map $\mathcal{Z}(D, r) \rightarrow \mathcal{X}_{0}(N)$ yields a map $C_{D} \rightarrow \mathcal{X}_{0}(N)$, still denoted $\mathbf{j}_{D}$. Note that this map also depends on the choice of $r$. For simplicity, we do not reflect this in the notation.

Fix $D_{0}, D_{1} \in \mathbb{Z}_{<0}$ and assume that they satisfy the properties of Definition 4.2. In particular, there are $r_{0}, r_{1} \in \mathbb{Z}$, such that $r_{i}^{2} \equiv D_{i} \bmod 4 N$. We write $\mathfrak{n}_{i}$ for the corresponding 
ideals of norm $N$ in $\mathcal{O}_{D_{i}}$ generated by $N$ and $\frac{r_{i}+\sqrt{D_{i}}}{2}$. Moreover, assume that $D_{0}$ is a fundamental discriminant with $D_{0} \equiv 1 \bmod 4$ and $D_{0} D_{1}$ is not a perfect square so that $\mathcal{Z}\left(D_{0}, r_{0}\right)$ and $\mathcal{Z}\left(D_{1}, r_{1}\right)$ intersect properly.

For a $C M$ elliptic curve $(E, \iota) \in C_{D_{0}}(S)$, we define

$$
\mathcal{O}_{E, \mathfrak{n}_{0}}:=\operatorname{End}_{S}\left(E \rightarrow E / E\left[\mathfrak{n}_{0}\right]\right)=\left\{\alpha \in \operatorname{End}_{S}(E) \mid \pi \alpha \pi^{-1} \in \operatorname{End}_{S}\left(E / E\left[\mathfrak{n}_{0}\right]\right)\right\} .
$$

We are interested in the intersection of $\mathcal{Z}\left(D_{0}, r_{0}\right)$ and $\mathcal{Z}\left(D_{1}, r_{1}\right)$ on $\mathcal{X}_{0}(N)$ or, equivalently, in the pullback of $Z\left(D_{1}, r_{1}\right)$ under $\mathbf{j}_{D_{0}}$. The stack $\mathbf{j}_{D_{0}}^{*} \mathcal{Z}\left(D_{1}, r_{1}\right)$ represents the following moduli problem: For a base scheme $S$, consider the category $\mathcal{M}\left(D_{1}, r_{1}, \mathfrak{n}_{0}\right)(S)$ of triples $(E, \iota, \phi)$, where

(i) $(E, \iota) \in C_{D_{0}}(S)$,

(ii) $\phi: \mathcal{O}_{D_{1}} \hookrightarrow \mathcal{O}_{E, \mathfrak{n}_{0}}$ is an action of $\mathcal{O}_{D_{1}}$, such that

(iii) $\phi\left(\mathfrak{n}_{1}\right) E\left[\mathfrak{n}_{0}\right]=0$.

We consider the fiber product diagram

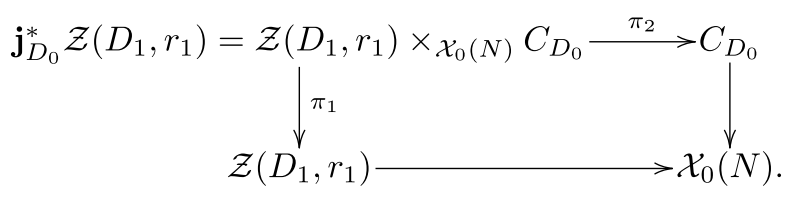

Lemma 5.2 The map

$$
\varphi: \mathbf{j}_{D_{0}}^{*} \mathcal{Z}\left(D_{1}, r_{1}\right) \rightarrow \mathcal{M}\left(D_{1}, r_{1}, \mathfrak{n}_{0}\right)
$$

given by

$$
\xi \mapsto(E, \iota, \phi),
$$

where $\pi_{2}(\xi)=(E, \iota)$ and $\pi_{1}(\xi)=\left(E \rightarrow E / E\left[\mathfrak{n}_{0}\right], \phi\right)$ is an isomorphism of stacks.

Proof This can be found in [6, Section 7.3]. It is clear that the described map is well defined and injective over any scheme $S$. In the other direction, suppose that $(E, \iota, \phi) \in \mathcal{M}\left(D_{1}, r_{1}, \mathfrak{n}_{0}\right)(S)$. Then $(E, \iota) \in C_{D_{0}}(S)$ and $\left(E \rightarrow E / E\left[\mathfrak{n}_{0}\right], \phi\right) \in \mathcal{Z}\left(D_{1}, r_{1}\right)(S)$ by definition. Thus, we obtain maps $\mathcal{M}\left(D_{1}, r_{1}, \mathfrak{n}_{0}\right) \rightarrow \mathcal{Z}\left(D_{1}, r_{1}\right)$ and $\mathcal{M}\left(D_{1}, r_{1}, \mathfrak{n}_{0}\right) \rightarrow C_{D_{0}}$. By the universal property of the fiber product, we obtain a unique map $\tilde{\varphi}$ that makes the following diagram commutative.

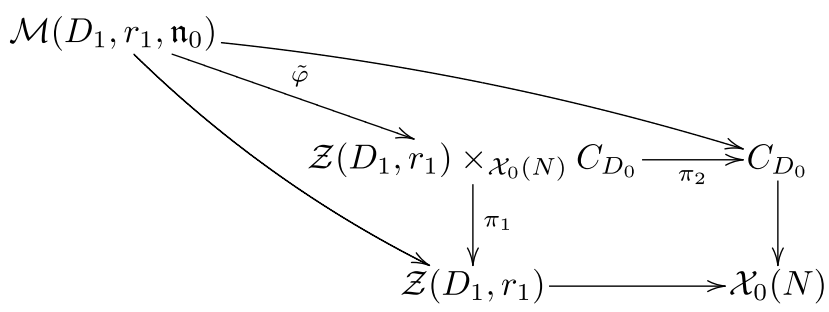

Therefore, we have $\xi=\tilde{\varphi}((E, \iota, \phi)) \in \mathbf{j}_{D_{0}}^{*} \mathcal{Z}\left(D_{1}, r_{1}\right)$ with $\varphi(\xi)=(E, \iota, \phi)$ by the definition of $\varphi$. 
Lemma 5.3 We have the identity

$$
\mathbf{j}_{D_{0}}^{*} \mathcal{Z}\left(D_{1}, r_{1}\right)=\sum_{\substack{n \equiv r_{0} r_{1} \bmod 2 N \\ n^{2} \leq D_{0} D_{1}}} \mathcal{Z}\left(\frac{D_{0} D_{1}-n^{2}}{4 N\left|D_{0}\right|}, \mathfrak{n}_{0}, \frac{n+r_{1} \sqrt{D_{0}}}{2 \sqrt{D_{0}}}\right)
$$

of divisors on $C_{D_{0}}$.

Proof This can be seen using Lemma 5.2. See Lemma 7.12 in [6], where the authors prove an identity on geometric points over algebraically closed fields in any characteristic. We also refer to [7] for details.

To ease the notation a bit, we continue to write $D=D_{0}$ and let $\rho \in \mathbb{Z}$, such that $\rho^{2} \equiv D \bmod 4 N$ (i.e. $\rho=r_{0}$ ). For a rational function $f \in \mathbb{Q}\left(\mathcal{X}_{0}(N)\right)$ we consider the pullback $\mathbf{j}_{D}^{*}\left(\left.f\right|_{\mathbf{j}_{D}\left(C_{D}\right)}\right)$, which makes sense as long as $\mathbf{j}_{D}\left(C_{D}\right)$ is not contained in the divisor of $f$. The element $\mathbf{j}_{D}^{*} f$ then defines an element of the function field $\mathbb{Q}\left(C_{D}\right) \cong \mathbb{Q}\left(\mathbf{C}_{D}\right)=H$, the Hilbert class field of $k_{D}=\mathbb{Q}(\sqrt{D})$.

Now let $f \in \mathbb{Q}\left(\mathcal{X}_{0}(N)\right)$ be a modular function such that its divisor is a linear combination of the Heegner divisors $\mathcal{Z}(m, \mu)$. That is, there are integers $c(m, r)$, such that

$$
\operatorname{div}(f)=\sum_{r \bmod 2 N} \sum_{\substack{d \in \mathbb{Q}_{<0} \\ d \equiv r^{2} \bmod 4 N}} c(d, r) \mathcal{Z}(d, r)+C(f),
$$

where $C(f)$ is supported at the boundary. We have by definition (cf. the proof of Proposition 3.7 in [29]) that

$$
\operatorname{div}\left(\mathbf{j}_{D}^{*} f\right)=\mathbf{j}_{D}^{*}(\operatorname{div}(f)) .
$$

Finally, we normalize the map pr: $C_{D} \rightarrow \operatorname{Spec} \mathcal{O}_{H}$ in the following way (it is only unique up to an automorphism of $\mathcal{O}_{H}$ ). We fix an embedding of $H$ into $\mathbb{C}$ and the integral ideal

$$
\mathfrak{n}=\left(N, \frac{\rho+\sqrt{D}}{2}\right) \subset \mathcal{O}_{D}
$$

of norm $N$. Consider the Heegner point $z_{D, \rho}$ given by $\pi: \mathbb{C} / \mathcal{O}_{D} \rightarrow \mathbb{C} / \mathfrak{n}^{-1}$. Then we require that $\mathrm{pr}$ is chosen such that $\operatorname{pr}_{*} \mathbf{j}^{*}\left(f^{w_{k}}\right) \in H$ is equal to $f\left(z_{D, \rho}\right)$, where $w_{k}$ is the number of roots of unity in $k=k_{D}$. As a point in $\mathbb{H}$, we can take $z_{D, \rho}$, such that its image under the Fricke involution is

$$
\frac{-1}{N z_{D, \rho}}=\frac{-\rho+\sqrt{D}}{2 N} \in \mathbb{H}
$$

according to our remark at the end of the last section. Note that $f\left(z_{D, \rho}\right) \in H$ defines a divisor on $\operatorname{Spec} \mathcal{O}_{H}$ given by

$$
\sum_{\mathfrak{P} \subset \mathcal{O}_{H}} \operatorname{ord}_{\mathfrak{P}}\left(f\left(z_{D, \rho}\right)\right) \mathfrak{P} .
$$


Here, the sum is over all nonzero prime ideals of $\mathcal{O}_{H}$.

Theorem 5.4 Let $D<0$ be an odd fundamental discriminant, $\rho \in \mathbb{Z}$ with $\rho^{2} \equiv D \bmod 4 N$ and normalize $\operatorname{pr}$ as described above. Suppose that $f \in \mathbb{Q}\left(\mathcal{X}_{0}(N)\right)$ with

$$
\operatorname{div}(f)=\sum_{r \bmod 2 N} \sum_{\substack{d \in \mathbb{Z}_{<0} \\ d \equiv r^{2} \bmod 4 N}} c(d, r) \mathcal{Z}(d, r)+C(f),
$$

where $C(f)$ is supported at the cusps and $\operatorname{div}(f)$ and $\mathcal{Z}(D, \rho)$ intersect properly. Then we have

$$
\operatorname{ord}_{\mathfrak{P}}\left(f\left(z_{D, \rho}\right)\right)=w_{k} \sum_{r \bmod 2 \mathrm{~N}} \sum_{d \in \mathbb{Z}_{<0}} c(d, r) \sum_{\substack{n \equiv \rho \cdot r \bmod 2 N \\ n^{2} \leq d D}} \mathcal{Z}\left(\frac{d D-n^{2}}{4 N|D|}, \mathfrak{n}, \frac{n+r \sqrt{D}}{2 \sqrt{D}}\right)_{\mathfrak{P}}
$$

for every prime $\mathfrak{P}$ of the Hilbert class field $H$.

Proof This follows directly from our considerations above and the fact that the pullback of $\operatorname{div}(f)$ as a Cartier divisor (8) agrees with the pullback as a Weil divisor, corrected by the multiplicity as explained above. The pullback as a Weil divisor is described in Lemma 5.3. Also note that the image of $C_{D}$ does not intersect the boundary.

Remark 5.5 The formulas in Propositions 3.8 and 3.9 provide explicit formulas for the quantities in Theorem 5.4.

We now recover the Theorem of Gross and Zagier on singular moduli [3] and its generalization by Dorman [4]. Let $D$ be a negative fundamental discriminant and suppose that $D$ is odd. Moreover, let $d$ be a negative discriminant, coprime to $D$. As in "Background", we consider the modular function

$$
\Psi(z, d)=\prod_{Q \in \mathrm{SL}_{2}(\mathbb{Z}) \backslash \mathcal{Q}_{d}}\left(j(z)-j\left(\alpha_{Q}\right)\right)^{1 / w_{d}} .
$$

Here, $\mathcal{Q}_{d}$ is the set of quadratic forms of discriminant $d$ and $w_{d}$ is the number of roots of unity in $\mathbb{Q}(\sqrt{d})$.

Theorem 5.6 (Gross-Zagier, Dorman) Let $H$ be the Hilbert class field of $k=\mathbb{Q}(\sqrt{D})$. Moreover, let $L$ be the fixed field of $\operatorname{Gal}(H / k)[2]$, let $p$ be a rational prime and $\mathfrak{f} \subset \mathcal{O}_{L}$ be the prime ideal below $\mathfrak{P}_{0} \mid p$ (see "Special endomorphisms"). Then we have

$$
\operatorname{ord}_{\mathfrak{f}} \sigma\left(\Psi\left(z_{D, \rho}, d\right)\right)=\frac{w_{k}}{4} \sum_{\substack{n \in \mathbb{Z} \\ n \equiv 1 \bmod 2}} 2^{o(n)} \bar{v}_{p}\left(\frac{D d-n^{2}}{4|D|}\right) \rho\left(\frac{D d-n^{2}}{4 p},[\mathfrak{c}]^{-2}\left[\mathfrak{c}_{0}\right]\right)
$$

for $\sigma=\sigma(\mathfrak{c})$. Here, $\bar{v}_{p}(x)=v_{p}(x)$ if $\operatorname{Diff}(x)=\{p\}$ and $\bar{v}_{p}(x)=0$, otherwise.

To conclude this section and justify the simpler version (Theorem 1) of Theorem 5.4 in "Background", we show that there is a rather simple criterion to decide if the divisor of a 
modular function on $\mathcal{X}_{0}(N)$ is horizontal if $N$ is square-free. It is reflected in the classical Kronecker congruence modulo $p$ of the modular equation of level $N$.

Recall that the fiber of $\mathcal{X}_{0}(N)$ above $p \nmid N$ is smooth and irreducible. We write the valuation on the function field that is induced by such a fiber by $v_{p}$. For $p \mid N$, the fiber has two irreducible components that intersect at each supersingular point. The cuspidal sections $\infty$ and 0 always only intersect one of those two fibers. We denote by $v_{p, \infty}$ the valuation on the function field of $\mathcal{X}_{0}(N)$ induced by the component above $p$ that intersects the cusp $\infty$ and by $v_{p, 0}$ the valuation corresponding to the other component.

For a modular function $f \in \mathbb{Q}\left(X_{0}(N)\right)=\mathbb{Q}\left(j, j_{N}\right)$ we denote by

$$
f_{\infty}(\tau)=\sum_{n \gg-\infty} c_{\infty}(n) q^{n}
$$

and

$$
f_{0}(\tau)=f_{\infty}\left(\frac{-1}{\tau}\right)=\sum_{n \gg-\infty} c_{0}(n) q^{n / N}
$$

the Fourier expansions of $f$ at the cusps $\infty$ and 0 , respectively.

Proposition 5.7 Let $f \in \mathbb{Q}\left(j, j_{N}\right)$ be a modular function for $\Gamma_{0}(N)$ and assume that $N$ is square-free. Let $p$ be a prime and set $a=\inf \left\{\operatorname{ord}_{p} c_{\infty}(n)\right\}$ and $b=\inf \left\{\operatorname{ord}_{p} c_{0}(n)\right\}$.

(i) If $p \nmid N$, then $a=v_{p}(f)$.

(ii) If $p \mid N$ then $a=v_{p, \infty}(f)$ and $b=v_{p, 0}(f)$.

Proof Evaluation at the Tate curve $\overline{\mathcal{G}}_{m}^{q} / q^{\mathbb{Z}}$ (cf. $[35,40]$ ) over $\mathbb{Z}[[q]]$ gives a homomorphism $\tau_{\infty}: \operatorname{Spec} \mathbb{Z}[[q]] \rightarrow \mathcal{X}_{0}(N)$, and evaluation at the Tate curve $\overline{\mathcal{G}}_{m}^{q^{1 / N}} / q^{\mathbb{Z}}$ over $\mathbb{Z}\left[\left[q^{1 / N}\right]\right]$ accordingly $\tau_{0}: \operatorname{Spec} \mathbb{Z}\left[\left[q^{1 / N}\right]\right] \rightarrow \mathcal{X}_{0}(N)$.

Combining these maps with the geometric points given by $q \mapsto 0$ for $\tau_{\infty}$ and $q^{1 / N} \mapsto 0$ for $\tau_{0}$, we obtain the cuspidal sections $\operatorname{Spec} \mathbb{Z} \rightarrow \mathcal{X}_{0}(N)$ corresponding to the cusps $\infty$ and 0, respectively. The pullbacks $\tau_{\infty}^{*} f$ and $\tau_{0}^{*} f$ of $f \in \mathbb{Q}\left(\mathcal{X}_{0}(N)\right)$ are given by the Fourier expansion of $f$ at $\infty$ and 0 , respectively. The valuation at $p$ on $\mathbb{Q}((q))$ and $\mathbb{Q}\left(\left(q^{1 / N}\right)\right)$ is given by the canonical extension of the $p$-adic valuation on $\mathbb{Q}$. It agrees with the valuations given by $a$ and $b$ in the statement of the Proposition. If $p \nmid N$ they coincide since the fiber of $\mathcal{X}_{0}(N)$ above $p$ is irreducible in this case.

We refer to Theorem VI. 3.10 on page 163 and Corollary 3.12 in [35] for more details. Moreover, in Section 3.16, ibid., the case $\mathcal{X}_{0}(p)$ for a prime $p$ is discussed in more detail.

\section{Borcherds products}

We will finally sketch how to apply our results to Borcherds products on modular curves.

Let $N$ be a positive integer and consider the congruence subgroup $\Gamma_{0}(N) \subset \mathrm{SL}_{2}(\mathbb{Z})$. The modular curve $Y_{0}(N):=\Gamma_{0}(N) \backslash \mathbb{H}$ can be obtained as an orthogonal modular variety as follows: 
Consider the vector space $V:=\left\{x \in M_{2}(\mathbb{Q}) \mid \operatorname{tr}(x)=0\right\}$ and define the quadratic form by $Q(x)=-N \operatorname{det}(x)$. The corresponding bilinear form is $(x, y)=N \operatorname{tr}(x y)$. The space $(V, Q)$ has signature $(2,1)$.

The symmetric domain $\mathbb{D}$ of $\mathrm{SO}_{V}(\mathbb{R}) \cong \mathrm{SO}(2,1)$ can be identified with the Grassmannian of two-dimensional positive definite subspaces of $V(\mathbb{R})$. It is isomorphic to the complex upper half-plane $\mathbb{H}$ via

$$
z=x+i y \mapsto\left[\left(\begin{array}{cc}
z & -z^{2} \\
1 & -z
\end{array}\right)\right] \mapsto \mathbb{R} \Re\left(\begin{array}{cc}
z & -z^{2} \\
1 & -z
\end{array}\right) \oplus \mathbb{R} \mathfrak{I}\left(\begin{array}{cc}
z & -z^{2} \\
1 & -z
\end{array}\right) .
$$

The action of $\gamma \in \mathrm{SL}_{2}$ is explicitly given by

$$
\gamma \cdot\left(\begin{array}{cc}
z & -z^{2} \\
1 & -z
\end{array}\right)=(c z+d)^{2}\left(\begin{array}{cc}
\gamma z & -(\gamma z)^{2} \\
1 & -\gamma z
\end{array}\right)
$$

where $\gamma z$ is the action via linear fractional transformations on $\mathbb{H}$.

In $V$ we have the even lattice

$$
L=\left\{\left(\begin{array}{cc}
b & -\frac{a}{N} \\
c & -b
\end{array}\right) \mid a, b, c \in \mathbb{Z}\right\}
$$

The dual lattice of $L$ is given by

$$
L^{\prime}=\left\{\left(\begin{array}{cc}
\frac{b}{2 N} & -\frac{a}{N} \\
c & -\frac{b}{2 N}
\end{array}\right) \mid a, b, c \in \mathbb{Z}\right\}
$$

Note that the discriminant group $L^{\prime} / L$ is cyclic of order $2 N$ and we can identify the corresponding finite quadratic module with the group $\mathbb{Z} / 2 N \mathbb{Z}$ together with the quadratic form $x^{2} / 4 N$, valued in $\frac{1}{4 N} \mathbb{Z} / \mathbb{Z} \subset \mathbb{Q} / \mathbb{Z}$. We denote by $M_{k, L}^{!}$the space of vector valued weakly holomorphic modular forms of weight $k$ and representation $\rho_{L}$, the Weil representation associated with $L$.

Let $f \in M_{1 / 2, L}^{!}$with Fourier expansion

$$
f(\tau)=\sum_{\mu \in \mathbb{Z} / 2 N \mathbb{Z}} \sum_{m \in \mathbb{Q}} c_{f}(m, \mu) e(m \tau) \phi_{\mu}
$$

and $c_{f}(m, \mu) \in \mathbb{Z}$ for all $m \leq 0$ and all $\mu \in \mathbb{Z} / 2 N \mathbb{Z}$. Borcherds [41] showed that there is a meromorphic modular form $\Psi_{L}(z, f)$ of weight $c_{f}(0,0)$ for $\Gamma_{0}(N)$ with divisor

$$
\operatorname{div}\left(\Psi_{L}(z, f)\right)=Z(f)=\sum_{r \bmod 2 N} \sum_{m<0} c_{f}(m, r) Z(4 N m, r)
$$

on $Y_{0}(N)$. Moreover, $\Psi_{L}(z, f)$ has an infinite product expansion of the form

$$
\Psi_{L}(z, f)=e\left(\left(\rho_{f}, z\right)\right) \prod_{n=1}^{\infty}(1-e(n z))^{c_{f}\left(n^{2} / 4 N, n\right)},
$$

which converges for $\Im(z)$ large enough and where $\rho_{f}$ is the corresponding Weyl vector at the cusp $\infty$. We refer to Borcherds [41, Theorem 13.3] and Bruinier and Ono [42, Theorem 6.1] for details. 
Lemma 6.1 Let $f \in M_{1 / 2, L}^{!}(\mathbb{Z})$ with constant coefficient $c_{f}(0,0)=0$ and $c_{f}(m, \mu) \in \mathbb{Q}$ for all $m \in \mathbb{Q}$ and $\mu \in L^{\prime} / L$. Then there exists an integer $M_{f}$, such that the Borcherds product $\Psi_{L}\left(z, h, M_{f} \cdot f\right)$ defines a meromorphic modular function contained in $\mathbb{Q}\left(j, j_{N}\right)$.

Proof Since the Fourier coefficients of $f$ have bounded denominators, replacing $f$ by an integral multiple $f^{\prime}=M \cdot f$ we obtain only integral coefficients. We view $\Psi_{L}\left(z, h, f^{\prime}\right)$ as a meromorphic function on $X_{0}(N)$. The multiplier system of $\Psi\left(z, f^{\prime}\right)$ has finite order, which can be shown using the embedding trick ([41, Lemma 8.1], [43]). Together with the integrality of the $c_{f^{\prime}}(m, \mu)$ this implies that the Fourier expansions of $\Psi\left(z, f^{\prime}\right)$ have rational coefficients at all cusps (for square-free $N$ ). Thus, $\Psi\left(z, M^{\prime} f\right)$ for some $M^{\prime} \in \mathbb{Z}$ is contained in the field $\mathbb{Q}\left(X_{0}(N)\right)=\mathbb{Q}\left(j, j_{N}\right)$ by the $q$-expansion principle.

Theorem 6.2 Let $f \in M_{1 / 2, L}^{!}(\mathbb{Z})$ be a weakly holomorphic modular form with only integral Fourier coefficients and assume that $N$ is square-free. Suppose that the multiplier system of $\Psi_{L}(z, h, f)$ is trivial. Then the divisor of the rational function defined by $\Psi_{L}(z, h, f)$ on $\mathcal{Y}_{0}(N)$ is equal to $\mathcal{Z}(f)$, the flat closure of $Z(f)$.

Proof The triviality of the multiplier system implies that $\Psi(z):=\Psi_{L}(z, f) \in \mathbb{Q}\left(j, j_{N}\right)$, as we have seen. The product expansion implies that the Fourier expansion of $\Psi(z)$ at the cusp $\infty$ has coprime integral coefficients under our assumption on $f$. Therefore, the fibers for $p \nmid N$ cannot occur in the divisor.

The Fricke involution $W_{N}$ is contained in $\mathrm{GSpin}_{V}(\mathbb{Q})$ and its image belongs to $\mathrm{SO}^{+}(L)$ (cf. [42]). We denote by $\sigma_{N}$ the image of $W_{N}$ in $\mathrm{O}\left(L^{\prime} / L\right)$. It acts on $L^{\prime} / L$ as $\mu \mapsto-\mu$ and, therefore,

$$
-4 \log \left|\left(\Psi \mid W_{N}\right)(z)\right|=\Phi\left(\frac{-1}{N z}, f\right)=\Phi\left(z, f_{N}\right)=-4 \log \left|\Psi\left(z, f_{N}\right)\right|,
$$

where $f_{N}=f^{\sigma_{N}}$.

We have that $c_{f}(m, \mu)=c_{f}(m,-\mu)$ by the action of the center of $\mathrm{Mp}_{2}(\mathbb{Z})$. Therefore, $f_{N}=f$ and $\Psi \mid W_{N}(z)= \pm \Psi(z)$ because $W_{N}^{2}=1$. This implies that the Fourier expansion of $\Psi(z)$ at the cusp 0 has also coprime integral Fourier coefficients and the result follows by Proposition 5.7 .

Now let $z_{D, \rho}$ be the Heegner point of level $N$ given by

$$
z_{D, \rho}=\frac{\rho+\sqrt{D}}{2 N} \in \mathbb{H},
$$

where we assume as usual that $D<0$ is an odd fundamental discriminant. We conclude that if $z_{D, \rho}$ is not contained in the divisor of $\Psi_{L}(z, f)$, then the prime valuations of $\Psi_{L}\left(z_{D, \rho}, f\right)$ can be obtained using Theorem 5.4.

\section{Some numerical examples}

We give some examples to illustrate and test our formula numerically.

The Borcherds products discussed in the previous section provide an easy way to generate examples if the space of obstructions $S_{3 / 2, L^{-}}$is known. Here, $S_{3 / 2, L^{-}}$is the space of 
cusp forms transforming with the Weil representation $\rho_{L^{-}}$of the lattice given by $L$ with the negative $-Q$ of the associated quadratic form. According to [44], if $N=p$ is prime, then $S_{3 / 2, L^{-}}=\{0\}$ if and only if the modular curve $X_{0}^{+}(p)=\Gamma_{0}^{+}(p) \backslash \mathbb{H}$ has genus zero. Here, $\Gamma_{0}^{+}(p)$ is the extension of $\Gamma_{0}(p)$ by the Fricke involution.

As an explicit and non-trivial example of this kind let us take $N=47$. Since $S_{3 / 2, L^{-}}$ vanishes, there exists a weakly holomorphic modular form $f$ of weight $1 / 2$ with representation $\rho_{L}$ and principal part

$$
\frac{1}{2} q^{-11 / 188}\left(\phi_{41}+\phi_{-41}\right)
$$

We can assume that the constant term of $f$ vanishes. Its Borcherds lift can be identified as the Hauptmodul for $X_{0}^{+}(47)$ which has a Fourier development starting with

$$
\begin{aligned}
\Psi(z, f)= & q^{-1}+1+q+2 q^{2}+3 q^{3}+3 q^{4}+5 q^{5}+5 q^{6}+8 q^{7}+9 q^{8} \\
& +12 q^{9}+14 q^{10}+O\left(q^{11}\right) .
\end{aligned}
$$

It can be explicitly constructed as the quotient

$$
\frac{\theta_{1}(\tau)-\theta_{2}(\tau)}{2 \eta(\tau) \eta(47 \tau)}+1
$$

where $\theta_{i}(\tau)$ are the two theta functions corresponding to the binary quadratic forms $Q_{1}=[1,1,12]$ and $Q_{2}=[2,-1,6]$ of discriminant -47 . Its divisor is equal to $\frac{1}{2}(Z(11,41)+Z(11,-41))$ which equals $Z(11,41)$ on $X_{0}^{+}(47)$. Since it is the generator of the function field of $X_{0}^{+}(47)$, its values at CM points of fundamental discriminant generate the corresponding Hilbert class field. As it turns out, the class polynomials for $\Psi(z, f)$ have some advantages over the usual Hilbert class polynomials for the elliptic modular function $j(\tau)$. In particular, their discriminants and resultants are usually much smaller.

As a first example, take the $\mathrm{CM}$ points of discriminant -23 . Theorem 5.4 easily reveals that $\Psi(z, f)$ is a unit in the Hilbert class field for any such point (note that it cannot be equal to 0 ). In fact, it is easily confirmed numerically that $\Psi\left(z_{-23,27}, f\right)$ is a root of the polynomial $x^{3}-x^{2}+2 x-1$ with discriminant -23 . Using sage, we confirmed that these roots are indeed contained in the Hilbert class field of $\mathbb{Q}(\sqrt{-23})$ and any of these generates the Hilbert class field over $\mathbb{Q}(\sqrt{-23})$ (the polynomial is irreducible and since the constant coefficient is equal to 1 , the values are indeed units). To compare with the $j$-invariant, note that the Hilbert class polynomial for $j$ is equal to $x^{3}+3491750 x^{2}-5151296875 x+12771880859375$ and has discriminant $-1 \cdot 5^{18} \cdot 7^{12} \cdot 11^{4} \cdot 17^{2} \cdot 19^{2} \cdot 23$.

To see a non-unit, we can take for instance $D=-107$. In this case, we evaluate at the point $z_{-107,9}$. Numerically, we obtain $\Psi\left(z_{-107,9}, f\right) \approx 2.796321 \ldots$ This turns out to be the unique real root of $x^{3}-3 x^{2}+2 x-4$. We consult Theorem 5.4 together with Proposition 3.10 to obtain that all prime ideals that contribute to its prime ideal factorization lie above 2. Indeed, the only non-zero contribution in the Theorem comes from $n= \pm 7$ and $\operatorname{Diff}(6 / 107)=\{2\}$.

We fix the embedding of the Hilbert class field $H$ of $k=\mathbb{Q}(\sqrt{-107})$ into $\mathbb{C}$, such that $H=k(j)$ and $j$ is mapped to $j\left(\mathcal{O}_{k}\right) \approx-1.297832 \ldots \in \mathbb{R}$. Let $\mathfrak{P}_{0}, \mathfrak{P}_{1}$ and $\mathfrak{P}_{2}$ be the 
prime ideals of $H$ above 2 where $\mathfrak{P}_{0}$ is fixed by complex conjugation and $\overline{\mathfrak{P}_{1}}=\mathfrak{P}_{2}$. Since the two primes of $\mathbb{Q}(\sqrt{-107})$ above 3 are both not principal, the valuation at $\mathfrak{P}_{0}$ is equal to zero by Proposition 3.10. The valuation at the other two primes turn both out to be equal to $2 \cdot(1 / 4+1 / 4)=1$, which we can also confirm using sage.

\section{Acknowledgments}

This article contains some results that were contained in my thesis [7]. First and foremost I would like to thank my advisor Jan Hendrik Bruinier for his constant support and encouragement. I also thank Claudia Alfes and Tonghai Yang for their help. I also thank Benjamin Howard for helpful discussions and in particular for helping me with the proof of Proposition 2.5. Moreover, the helpful comments of the anonymous referee are greatly appreciated.

\section{Role of funding source}

This work is partially supported by DFG grant BR-2163/4-1.

\section{Compliance with ethical guidelines}

Competing interests

The author declares that he has no competing interests.

Received: 15 February 2015 Accepted: 11 May 2015

Published online: 17 August 2015

\section{References}

1. Weber, H.: Algebra, Dritter Band, 3rd edn. Chelsea Publishing Company, New York (1961)

2. Zagier, D.B.: Traces of singular moduli. In: Motives, Polylogarithms and Hodge Theory, Part I (Irvine, CA, 1998). Int. Press Lect. Ser., vol. 3, pp. 211-244. International Press, Somerville (2002)

3. Gross, B.H., Zagier, D.B.: On singular moduli. J. Reine Angew. Math. 355, 191-220 (1985). doi:10.1016/j.jnt.2006.04.009

4. Dorman, D.R.: Special values of the elliptic modular function and factorization formulae. J. Reine Angew. Math. 383 207-220 (1988). doi:10.1515/crll.1988.383.207

5. Gross, B.H., Kohnen, W., Zagier, D.B.: Heegner points and derivatives of L-series. II. Math. Ann. 278(1-4), 497-562 (1987). doi:10.1007/BF01458081

6. Bruinier, J.H., Yang, T.: Faltings heights of CM cycles and derivatives of L-functions. Invent. Math. 177(3), 631-681 (2009). doi:10.1007/s00222-009-0192-8

7. Ehlen, S.: CM Values of Regularized Theta Lifts. TU Darmstadt, Darmstadt (2013). http://tuprints.ulb.tu-darmstadt. de/3731/

8. Ehlen, S.: CM values of regularized theta lifts (preprint) (2015)

9. Kudla, S.S., Rapoport, M., Yang, T.: On the derivative of an Eisenstein series of weight one. Int. Math. Res. Notices 7 347-385 (1999). doi:10.1155/S1073792899000185

10. Borcherds, R.E.: The Gross-Kohnen-Zagier theorem in higher dimensions. Duke Math. J. 97(2), 219-233 (1999)

11. Bruinier, J.H.: On the converse theorem for Borcherds products. ArXiv:1210.4821 (e-prints) (2012).

12. Kudla, S.S., Rapoport, M., Yang, T.: Derivatives of Eisenstein series and Faltings heights. Compos. Math. 140(4), 887-951 (2004). doi:10.1112/S0010437X03000459

13. Koblitz, N.: Introduction to elliptic curves and modular forms, 2nd edn. In: Graduate Texts in Mathematics, vol. 97, p. 248. Springer, New York (1993)

14. Miyake, T.: Modular forms, English edn. In: Springer Monographs in Mathematics, p. 335. Springer, Berlin (2006) Translated from the 1976 Japanese original by Yoshitaka Maeda

15. Ono, K.: The web of modularity: arithmetic of the coefficients of modular forms and q-series. In: CBMS Regional Conference Series in Mathematics, vol. 102, p. 216. Published for the Conference Board of the Mathematical Sciences, Washington, DC (2004)

16. Stein, W.: Modular forms, a computational approach. In: Graduate Studies in Mathematics, vol. 79, p. 268. American Mathematical Society, Providence (2007). With an appendix by Paul E. Gunnells

17. Shimura, G.: Introduction to the arithmetic theory of automorphic functions. In: Publications of the Mathematical Society of Japan, vol. 11, p. 271. Princeton University Press, Princeton (1994). Reprint of the 1971 original, Kanô Memorial Lectures, 1

18. Katz, N.M., Mazur, B.: Arithmetic moduli of elliptic curves. In: Annals of Mathematics Studies, vol. 108, p. 514. Princeton University Press, Princeton (1985)

19. Silverman, J.H.: The arithmetic of elliptic curves, 2nd edn. In: Graduate Texts in Mathematics, vol. 106, p. 513. Springer, Dordrecht (2009). doi:10.1007/978-0-387-09494-6

20. Silverman, J.H.: Advanced topics in the arithmetic of elliptic curves. In: Graduate Texts in Mathematics, vol. 151, p. 525. Springer, New York (1994). doi:10.1007/978-1-4612-0851-8

21. Deuring, M.: Die Typen der Multiplikatorenringe elliptischer Funktionenkörper. Abh. Math. Sem. Univ. Hamburg 14(1), 197-272 (1941). doi:10.1007/BF02940746

22. Howard, B.: Rankin-Selberg L-functions and cycles on unitary Shimura varieties. http://www2.bc.edu/howardbe/ Research/barbados.pdf (2013)

23. Lan, K.-W.: Arithmetic compactifications of PEL-type shimura varieties, p. 1077. Ph.D. Thesis, Harvard University. ProQuest LLC, Ann Arbor (2008) 
24. Bruinier, J.H., Howard, B., Yang, T.: Heights of Kudla-Rapoport divisors and derivatives of L-functions. Invent. Math. ArXiv:1303.0549 (e-prints) (2013, to appear)

25. Howard, B.: Moduli spaces of CM elliptic curves. http://www2.bc.edu/howardbe/Research/morningside.pdf (2012)

26. Conrad, B.: Gross-Zagier revisited. In: Heegner Points and Rankin L-series. Math. Sci. Res. Inst. Publ., vol. 49, pp. 67-163. Cambridge University Press, Cambridge (2004). doi:10.1017/CBO9780511756375.006. With an appendix by W. R. Mann

27. Kudla, S.S., Yang, T.: On the pullback of an arithmetic theta function. Manuscripta Math. 140(3-4), 393-440 (2013). doi:10.1007/s00229-012-0569-7

28. Serre, J.-P.: A course in arithmetic. In: Graduate Texts in Mathematics, vol. 7, p. 115. Springer, New York (1973). Translated from the French

29. Vistoli, A.: Intersection theory on algebraic stacks and on their moduli spaces. Invent. Math. 97(3), 613-670 (1989). doi:10.1007/BF01388892

30. Ehlen, S.: Vector valued theta functions associated with binary quadratic forms. ArXiv:1505.02693 (preprint) (2015)

31. Dorman, D.R.: Global orders in definite quaternion algebras as endomorphism rings for reduced CM elliptic curves. Théorie des Nombres (Quebec. PQ, 1987), pp. 108-116. de Gruyter, Berlin (1989)

32. Zagier, D.B.: Zetafunktionen und Quadratische Köorper, p. 144. Springer, Berlin (1981). Eine Einführung in die höhere Zahlentheorie (An introduction to higher number theory). Hochschultext (University Text)

33. Serre, J.-P.: Local fields. In: Graduate Texts in Mathematics, vol. 67, p. 241. Springer, New York (1979). Translated from the French by Marvin Jay Greenberg

34. Serre, J.-P., Tate, J.: Good reduction of abelian varieties. Ann. Math. 2(88), 492-517 (1968)

35. Deligne, P., Rapoport, M.: Les schémas de modules de courbes elliptiques. In: Modular Functions of One Variable, II (Proceedings of International Summer School, University of Antwerp, Antwerp, 1972), pp. 143-316349. Springer, Berlin (1973)

36. Gross, B.H., Zagier, D.B.: Heegner points and derivatives of L-series. Invent. Math. 84(2), 225-320 (1986). doi:10.1007/ BF01388809

37. Conrad, B.: Arithmetic moduli of generalized elliptic curves. J. Inst. Math. Jussieu 6(2), 209-278 (2007). doi:10.1017/ S1474748006000089

38. Birch, B.J.: Heegner points of elliptic curves. In: Symposia Mathematica, vol. XV (Convegno di Strutture in Corpi Algebrici, INDAM, Rome, 1973), pp. 441-445. Academic Press, London (1975)

39. Gross, B.H.: Heegner points on Xo(N). In: Modular Forms (Durham, 1983). Ellis Horwood Ser. Math. Appl.: Statist. Oper. Res., pp. 87-105. Horwood, Chichester (1984)

40. Diamond, F., Im, J.: Modular forms and modular curves. In: Seminar on Fermat's Last Theorem (Toronto, ON, 1993-1994). CMS Conference Proceedings, vol. 17, pp. 39-133. American Mathematical Society, Providence (1995)

41. Borcherds, R.E.: Automorphic forms with singularities on Grassmannians. Invent. Math. 132 (3), 491-562 (1998)

42. Bruinier, J., Ono, K.: Heegner divisors, L-functions and harmonic weak Maass forms. Ann. Math. 2 172(3), 2135-2181 (2010). doi:10.4007/annals.2010.172.2135

43. Borcherds, R.E.: Correction to: "The Gross-Kohnen-Zagier theorem in higher dimensions" [Duke Math. J. 97 (1999), no. 2, 219-233]. Duke Math. J. 105(1), 183-184 (2000). doi:10.1215/S0012-7094-00-10519-4

44. Bruinier, J.H., Ehlen, S., Freitag, E.: Lattices with many Borcherds products. ArXiv:1408.4148 (e-prints) (2014)

\section{Submit your manuscript to a SpringerOpen ${ }^{\circ}$ journal and benefit from:}

- Convenient online submission

- Rigorous peer review

- Immediate publication on acceptance

- Open access: articles freely available online

- High visibility within the field

- Retaining the copyright to your article

Submit your next manuscript at $\boldsymbol{\nabla}$ springeropen.com 